\title{
Investigating the Covering Fraction Distribution of Swift/BAT AGNs with X-Ray and Infrared Observations
}

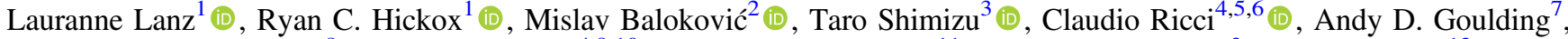 \\ David R. Ballantyne ${ }^{8}$ (D), Franz E. Bauer ${ }^{4,9,10}$ (ID), Chien-Ting J. Chen ${ }^{11}$ (D), Agnese del Moro ${ }^{3}$, Duncan Farrah ${ }^{12}$ (D), \\ Michael, J. Koss ${ }^{13}$ (D), Stephanie LaMassa ${ }^{14}$ (D), Alberto Masini ${ }^{1,15,16}$ (D), and Luca Zappacosta ${ }^{17}$ (D) \\ ${ }^{1}$ Department of Physics and Astronomy, Dartmouth College, 6127 Wilder Laboratory, Hanover, NH 03755, USA \\ ${ }^{2}$ Harvard-Smithsonian Center for Astrophysics, 60 Garden Street, Cambridge, MA 02138, USA \\ ${ }^{3}$ Max-Planck Institute for Extra-terrestrial Physics, Giessenbachstrasse 1, D-85741 Garshing, Germany \\ ${ }^{4}$ Núcleo de Astronomía de la Falcultad de Ingeniería, Unversidad Diego Potales, Av. Ejército Libertador 441, Santiago, Chile \\ ${ }^{5}$ Kavli Institute for Astronomy and Astrophysics, Peking University, Beijing 100871, People's Republic of China \\ ${ }^{6}$ Chinese Academy of Sciences South America Center for Astronomy, Camino El Observatorio 1515, Las Condes, Santiago, Chile \\ ${ }^{7}$ Department of Astrophysics, Princeton University, Princeton, NJ 08544, USA \\ ${ }^{8}$ Center for Relativistic Astrophysics, School of Physics, Georgia Institute of Technology, 837 State Street, Atlanta, GA 30332, USA \\ ${ }^{9}$ Millennium Institute of Astrophysics (MAS), Nuncio Monseñor Sótero Sanz 100, Providencia, Santiago, Chile \\ ${ }^{10}$ Space Science Institute, 4750 Walnut Street, Suite 205, Boulder, Colorado 80301, USA \\ ${ }^{11}$ Department of Astronomy and Astrophysics, Pennsylvania State University, 514 Davey Lab, University Park, PA 16802, USA \\ ${ }_{12}$ Department of Physics, Virginia Tech, 850 West Campus Drive, Blacksburg, VA 24061, USA \\ 13 Eureka Scientific Inc., 2542 Delmar Avenue, Suite 100, Oakland, CA, 94602-3017, USA \\ ${ }_{15}^{14}$ Space Telescope Science Institute, 3700 San Martin Dr., Baltimore, MD 21218, USA \\ ${ }^{15}$ INAF-Osservatorio Astronomico di Bologna, via Gobetti 93/3, I-40129 Bologna, Italy \\ ${ }^{16}$ Dipartimento di Fisica e Astronomia (DIFA), Universita di Bologna, via Gobetti 93/2, I-40129 Bologna, Italy \\ ${ }^{17}$ INAF-Osservatorio Astronomico di Roma, via di Frascati 33, I-00078 Monte Porzio Catone, Italy \\ Received 2017 December 22; revised 2018 October 25; accepted 2018 November 2; published 2018 December 31
}

\begin{abstract}
We present an analysis of a sample of 69 local obscured Swift/Burst Alert Telescope active galactic nuclei (AGNs) with X-ray spectra from NuSTAR and infrared (IR) spectral energy distributions from Herschel and WISE. We combine this X-ray and IR phenomenological modeling and find a significant correlation between reflected hard $\mathrm{X}$-ray emission and IR AGN emission, with suggestive indications that this correlation may be stronger than the one between intrinsic hard X-ray and IR emissions. This relation between the IR and reflected X-ray emission suggests that both are the result of the processing of intrinsic emission from the corona and accretion disk by the same structure. We explore the resulting implications on the underlying distribution of covering fraction for all AGNs, by generating mock observables for the reflection parameter and IR luminosity ratio using empirical relations found for the covering fraction with each quantity. We find that the observed distributions of the reflection parameter and IR-to-X-ray ratio are reproduced with broad distributions centered around covering fractions of at least $\sim 40 \%-50 \%$, whereas narrower distributions match our observations only when centered around covering fractions of $\sim 70 \%-80 \%$. Our results are consistent with both independent estimates of the covering fractions of individual objects and the typical covering fraction obtained on the basis of obscured fractions for samples of AGNs. These results suggest that the level of reprocessing in AGNs, including X-ray reflection, is related in a relatively straightforward way to the geometry of the obscuring material.
\end{abstract}

Key words: galaxies: active - galaxies: nuclei - galaxies: Seyfert - X-rays: galaxies - infrared: galaxies

\section{Introduction}

Understanding the role of supermassive black holes (SMBHs) in the evolution of galaxies remains one of the pressing open questions in astronomy (e.g., Kormendy \& Ho 2013; Hickox \& Alexander 2018). There exist a number of lines of evidence supporting the coevolution of SMBHs and their host galaxies (e.g., Bell et al. 2004; Gültekin et al. 2009; Oppenheimer et al. 2010; McConnell \& Ma 2013). Most of an SMBH's growth is thought to occur during its active phases (e.g., Marconi et al. 2004; Merloni \& Heinz 2008; Alexander \& Hickox 2012). Furthermore, active galactic nuclei (AGNs) provide the best stage for studying all but the most local SMBHs (e.g., $d \lesssim 50 \mathrm{Mpc}$, Xie $\&$ Yuan 2017), because it is during these phases that the nuclear regions emit the most radiation due to larger rates of gas accretion.

AGNs emit across most of the electromagnetic spectrum with a significant portion of the emission in the infrared (IR) at $1-100 \mu \mathrm{m}$ (e.g., Antonucci 1993; Efstathiou \& Rowan-Robinson 1995; Elitzur 2008; Padovani et al. 2017). The IR emission is thought to be due to a dusty "torus" (e.g., Krolik \& Begelman 1986; Netzer 2015), which is primarily heated as a result of the absorption of the optical and ultraviolet (UV) emission from the accretion disk. At X-ray energies, including in the $3-79 \mathrm{keV}$ range probed by the Nuclear Spectroscopic Telescope Array (NuSTAR; Harrison et al. 2013), the observed emission is due primarily to the corona above the disk. This wavelength regime, therefore, provides a window into the intrinsic emission very near to the AGNs, in part seen in the tight relationship that has been found between coronal and disk emission (e.g., Steffen et al. 2006; Lusso \& Risaliti 2017). Therefore, we might expect to also find a relationship between reprocessed UV emission, captured by thermal IR emission, and X-ray emission reprocessed primarily via absorption and reflection (e.g., Guilbert \& Rees 1988; Madau et al. 1993; Matt \& Fabian 1994). The main spectral signatures of reflection include both a hump in the 10-30 keV range due to Compton scattering and the Fe K $\alpha$ line (e.g., George \& Fabian 1991), whose narrow core peaking at $6.4 \mathrm{keV}$ provides strong evidence for interaction with 
cold material (e.g., Nandra et al. 1997; Reeves 2003; Levenson et al. 2006).

Together, the X-ray and IR emission allow us to probe the nature of the structure that reprocesses nuclear emission. In particular, the degree of clumpiness in this structure (e.g., Fritz et al. 2006; Nenkova et al. 2008), the relation of its properties to the AGN luminosity, and the distribution of its covering fraction for the AGN population are among the aspects of this structure that are still not completely understood. This last aspect is still poorly constrained both for all AGNs and for only the subset of obscured AGNs and typically examined using complex spectral models (e.g., Murphy \& Yaqoob 2009). Yaqoob \& Murphy (2011) examined the dependence of the IRto-X-ray luminosity ratio on other model parameters in one of these torus models, specifically MYTorus. They found that the ratio was relatively insensitive to column density and instead depended much more strongly on covering fraction and shape of the X-ray continuum.

Previous studies have found tight correlations between continuum mid-IR (MIR) and intrinsic soft X-ray $(<10 \mathrm{keV})$ luminosities of AGNs (e.g., Lutz et al. 2004; Gandhi et al. 2009; Asmus et al. 2011, 2015; Chen et al. 2017). The absence of a dependence on obscuring column depth in these relations does not meet the expectations of the classical torus models (Pier \& Krolik 1993). These classical models assume smooth and homogeneous dust distributions and predict a higher amount of obscuration for higher inclinations, resulting in an expected dependence of the reprocessed-to-intrinsic emission ratio on the obscuring column. In contrast, clumpy torus models invoke highly inhomogeneous gas, allowing for unobscured lines of sight even in edge-on configurations (e.g., Nikutta et al. 2009; Elitzur 2012; Stalevski et al. 2016). As a result, the clumpiness dilutes the dependence of the reprocessed-to-intrinsic emission ratio on orientation (e.g., Nenkova et al. 2008; Hönig \& Kishimoto 2010).

The Swift/Burst Alert Telescope (BAT; Gehrels et al. 2004; Barthelmy et al. 2005) on the Neil Gehrels Swift Observatory, operating at 14-195 keV, created the most sensitive hard X-ray survey of the entire sky. Its high energy range is well suited for penetrating large obscuring columns to detect AGNs with very little contamination from other host galaxy emission mechanisms (e.g., Koss et al. 2016). The soft X-ray properties of Swift/ BAT AGN have been studied in detail by several studies (e.g., Winter et al. 2009; Ricci et al. 2017a). Recently, large subsets from the Swift/BAT 58 month and 70 month AGN catalogs have been observed and analyzed separately in the near-IR (NIR), MIR, and far-IR (FIR; Meléndez et al. 2014; Lamperti et al. 2017; Shimizu et al. 2016, 2017, hereafter S17) and with detailed hard X-ray spectra taken by NUSTAR (Baloković et al. 2018, in preparation, hereafter B18).

Most analyses to date that have jointly used IR and hard X-ray observations of this unbiased sample of local AGNs have primarily explored the connections of the total observed NIR, MIR, and FIR emission, colors, and emission-line properties to the hard X-ray luminosities (e.g., Mushotzky et al. 2008; Diamond-Stanic et al. 2009; Rigby et al. 2009; Vasudevan et al. 2010; Matsuta et al. 2012; Ichikawa et al. 2012, 2017). However, a joint analysis using a detailed decomposition of the IR spectral energy distribution (SED) combined with good quality spectra extending into the hard X-ray regime has not yet been done for such samples of AGNs. ${ }^{18}$ In this article, we

\footnotetext{
18 This type of analysis has been performed for individual objects (e.g., Farrah et al. 2016)
}

combine the SED decompositions performed by Shimizu et al. (2017, S17) with the NuSTAR spectral analyses of Baloković et al. 2018 (in preparation, B18) of obscured AGNs to constrain the structure of the torus from purely phenomenological modeling. Our sample is one of the largest sample of obscured AGNs with detailed determination of their IR and hard X-ray properties.

This article is organized as follows. In Section 2, we describe the sample selection, followed by a summary of the data reduction and parameter extraction undertaken (Section 3). In Section 4, we discuss our analysis and modeling, as well as the resulting implications, and we summarize our conclusions in Section 5. Throughout this article, we assume a cosmology with Hubble constant $H_{0}=70 \mathrm{~km} \mathrm{~s}^{-1} \mathrm{Mpc}^{-1}$, matter density parameter $\Omega_{\mathrm{M}}=0.3$, and dark energy density $\Omega_{\Lambda}=0.7$ (Spergel et al. 2007). Unless otherwise specifically stated, uncertainties are $1 \sigma$ errors.

\section{Sample}

The sample presented in this work is the overlap of two other subsamples of the Swift/BAT 58 month and 70 month catalogs $^{2,3}$ (Baumgartner et al. 2013), specifically the Herschel sample of $\mathrm{S} 17$ and the NUSTAR sample of B18. The S17 sample is composed of 313 Swift/BAT AGNs at $z<0.05$ that are not blazars or BL Lac objects selected from the 58 month Swift/BAT catalog. ${ }^{19}$ It contains an approximately even mix of Seyfert types, based on optical spectra. A small fraction of S17 samples $(<5 \%)$ are unclassified AGNs or have low ionization nuclear emission-line region nuclei. The entire sample was observed with Herschel in five bands at $70 \mu \mathrm{m}, 160 \mu \mathrm{m}$, $250 \mu \mathrm{m}, 350 \mu \mathrm{m}$, and $500 \mu \mathrm{m}$.

We cross-correlated the S17 sample with the subset of the B18 sample at $z<0.05$, which contains 95 AGNs selected from the 70 month Swift/BAT catalog ${ }^{20}$ to have $14-195 \mathrm{keV}$ flux greater than $1 \times 10^{-11} \mathrm{erg} \mathrm{s}^{-1} \mathrm{~cm}^{-2}$ and be identified as a narrow-line AGN (i.e., Sy1.8, Sy1.9, or Sy2) ${ }^{21}$ in that catalog. They were all observed simultaneously with short NuSTAR and Swift/X-ray Telescope (XRT; Burrows et al. 2005) observations, typically $20 \mathrm{ks}$ and $7 \mathrm{ks}$, respectively. Sources with complex spectra (requiring models with multiple additional components beyond those described in Section 3.1) ${ }^{22}$ or low signal-to-noise spectra $(\lesssim 300$ counts) were also excluded from the B18 sample for greater uniformity in the quality of the $\mathrm{X}$-ray spectral analysis.

There are 69 AGNs in common to these two samples, which we use for our analysis in this work. Their names and coordinates are given in Table 1. This sample is one of the largest of obscured AGN with this high quality of IR and hard X-ray data. Given the varied selection criteria of the S17 and B18 samples and our combination thereof, we investigated how well each of them, as well as our overlap sample of 69 , is representative of the full Swift/BAT AGN sample (excluding

\footnotetext{
19 https://swift.gsfc.nasa.gov/results/bs58mon

20 https://swift.gsfc.nasa.gov/results/bs70mon

21 There has been some work suggesting that late intermediate Seyfert types (Sy1.8, Sy1.9) are more similar to unobscured AGNs (e.g., Stern \& Laor 2012; Hernández-García et al. 2017), although Koss et al. (2017) recently showed that Sy1.9 AGNs could have column densities up to the Compton-thick regime. Only 12 sources in our sample fall into this category and they are not clustered in the parameters we examine. As such, we do not believe their inclusion biases our conclusions.

${ }^{22}$ The five AGNs excluded for this reason are the Circinus Galaxy, NGC 424, NGC 1068, NGC 1192, and NGC 4945.
} 
Table 1

IR Parameters

\begin{tabular}{|c|c|c|c|c|}
\hline \multirow[b]{2}{*}{ Name } & \multirow[b]{2}{*}{$\begin{array}{l}\text { R.A. } \\
\text { (J2000) }\end{array}$} & \multirow[b]{2}{*}{$\begin{array}{l}\text { Decl. } \\
(\mathrm{J} 2000)\end{array}$} & \multicolumn{2}{|c|}{ IR Parameters } \\
\hline & & & $\begin{array}{l}\text { AGN Slope } \\
\qquad(\alpha)\end{array}$ & $\begin{array}{c}\text { Turnover } \\
\text { Wavelength }\left(\lambda_{\mathrm{C}}\right)\end{array}$ \\
\hline LEDA136991 & $00^{\mathrm{h}} 25^{\mathrm{m}} 32^{\mathrm{s}} .87$ & $+68^{\mathrm{d}} 21^{\mathrm{m}} 44^{\mathrm{s}} .2$ & $1.5_{-0.40}^{+0.53}$ & $45.40_{-15.9}^{+16.3}$ \\
\hline NGC262 & $00^{\mathrm{h}} 48^{\mathrm{m}} 47^{\mathrm{s}} .14$ & $+31^{\mathrm{d}} 57^{\mathrm{m}} 25^{\mathrm{s}} \cdot 1$ & $1.6_{-0.45}^{+0.52}$ & $43.16_{-12.9}^{+13.0}$ \\
\hline ESO 195-IG021 & $01^{\mathrm{h}} 00^{\mathrm{m}} 36^{\mathrm{s}} .53$ & $-47^{\mathrm{d}} 52^{\mathrm{m}} 02^{\mathrm{s}} .7$ & $1.7_{-0.32}^{+0.40}$ & $57.95_{-15.5}^{+16.8}$ \\
\hline IC 1663 & $01^{\mathrm{h}} 14^{\mathrm{m}} 07^{\mathrm{s}} .02$ & $-32^{\mathrm{d}} 39^{\mathrm{m}} 03^{\mathrm{s}} .2$ & $2.9_{-0.21}^{+0.30}$ & $67.68_{-9.27}^{+8.65}$ \\
\hline NGC513 & $01^{\mathrm{h}} 24^{\mathrm{m}} 26^{\mathrm{s}} .85$ & $+33^{\mathrm{d}} 47^{\mathrm{m}} 58^{\mathrm{s}} \cdot 0$ & $1.1_{-0.43}^{+0.54}$ & $43.52_{-15.4}^{+19.7}$ \\
\hline MCG-01-05-047 & $01^{\mathrm{h}} 52^{\mathrm{m}} 49^{\mathrm{s}} .00$ & $-03^{\mathrm{d}} 26^{\mathrm{m}} 48^{\mathrm{s}} \cdot 6$ & $2.0_{-0.23}^{+0.25}$ & $74.65_{-11.6}^{+12.1}$ \\
\hline NGC788 & $02^{\mathrm{h}} 01^{\mathrm{m}} 06^{\mathrm{s}} .45$ & $-06^{\mathrm{d}} 48^{\mathrm{m}} 55^{\mathrm{s}} .9$ & $1.7_{-0.30}^{+0.38}$ & $52.66_{-7.20}^{+8.73}$ \\
\hline NGC1052 & $02^{\mathrm{h}} 41^{\mathrm{m}} 04^{\mathrm{s}} .80$ & $-08^{\mathrm{d}} 15^{\mathrm{m}} 20.7$ & $1.6_{-0.27}^{+0.35}$ & $58.77_{-9.00}^{+12.1}$ \\
\hline 2MFGC 2280 & $02^{\mathrm{h}} 50^{\mathrm{m}} 42^{\mathrm{s}} .59$ & $+54^{\mathrm{d}} 42^{\mathrm{m}} 17^{\mathrm{s}} .6$ & $1.7_{-0.47}^{+0.64}$ & $43.73_{-16.7}^{+23.3}$ \\
\hline NGC1365 & $03^{\mathrm{h}} 33^{\mathrm{m}} 36^{\mathrm{s}} .37$ & $-36^{\mathrm{d}} 08^{\mathrm{m}} 25^{\mathrm{s}} .4$ & $2.1_{-0.41}^{+0.41}$ & $54.32_{-14.8}^{+11.6}$ \\
\hline 2MASXJ04234080+0408017 & $04^{\mathrm{h}} 23^{\mathrm{m}} 40^{\mathrm{s}} .77$ & $+04^{\mathrm{d}} 08^{\mathrm{m}} 01 . \mathrm{s} .8$ & $1.7_{-0.30}^{+0.45}$ & $50.48_{-12.3}^{+28.2}$ \\
\hline CGCG420-015 & $04^{\mathrm{h}} 53^{\mathrm{m}} 25^{\mathrm{s}} .75$ & $+04^{\mathrm{d}} 03^{\mathrm{m}} 41^{\mathrm{s}} .7$ & $1.7_{-0.51}^{+0.51}$ & $41.75_{-7.76}^{+1.1}$ \\
\hline ESO 033-G002 & $04^{\mathrm{h}} 55^{\mathrm{m}} 58^{\mathrm{s}} .96$ & $-75^{\mathrm{d}} 32^{\mathrm{m}} 28^{\mathrm{s}} \cdot 2$ & $1.5_{-0.33}^{+0.40}$ & $52.95_{-11.4}^{+14.2}$ \\
\hline LEDA178130 & $05^{\mathrm{h}} 05^{\mathrm{m}} 45^{\mathrm{s}} .73$ & $-23^{\mathrm{d}} 51^{\mathrm{m}} 14^{\mathrm{s}} .0$ & $1.6_{-0.35}^{+0.46}$ & $52.21_{-9.95}^{+13.7}$ \\
\hline 2MASXJ05081967+1721483 & $05^{\mathrm{h}} 08^{\mathrm{m}} 19^{\mathrm{s}} \cdot 69$ & $+17^{\mathrm{d}} 21^{\mathrm{m}} 48^{\mathrm{s}} .1$ & $2.1_{-0.39}^{+0.49}$ & $50.01_{-18.2}^{+20.3}$ \\
\hline NGC2110 & $05^{\mathrm{h}} 52^{\mathrm{m}} 11^{\mathrm{s}} .38$ & $-07^{\mathrm{d}} 27^{\mathrm{m}} 22.3$ & $1.4_{-0.42}^{+0.56}$ & $44.91_{-17.3}^{+21.2}$ \\
\hline ESO 005-G004 & $06^{\mathrm{h}} 05^{\mathrm{m}} 41^{\mathrm{s}} \cdot 63$ & $-86^{\mathrm{d}} 37^{\mathrm{m}} 54^{\mathrm{s}} .7$ & $1.0_{-0.38}^{+0.48}$ & $51.55_{-19.0}^{+18.5}$ \\
\hline ESO 121-IG028 & $06^{\mathrm{h}} 23^{\mathrm{m}} 45^{\mathrm{s}} .57$ & $-60^{\mathrm{d}} 58^{\mathrm{m}} 44^{\mathrm{s}} .4$ & $1.7_{-0.48}^{+0.64}$ & $39.81_{-13.1}^{+15.6}$ \\
\hline MCG $+06-16-028$ & $07^{\mathrm{h}} 14^{\mathrm{m}} 03^{\mathrm{s}} .86$ & $+35^{\mathrm{d}} 16^{\mathrm{m}} 45^{\mathrm{s}} \cdot 4$ & $1.4_{-0.41}^{+0.54}$ & $42.86_{-13.6}^{+19.1}$ \\
\hline LEDA96373 & $07^{\mathrm{h}} 26^{\mathrm{m}} 26^{\mathrm{s}} .35$ & $-35^{\mathrm{d}} 54^{\mathrm{m}} 21^{\mathrm{s}} .7$ & $2.0_{-0.39}^{+0.46}$ & $49.08_{-7.22}^{+11.2}$ \\
\hline UGC3995A & $07^{\mathrm{h}} 44^{\mathrm{m}} 06^{\mathrm{s}} .97$ & $+29^{\mathrm{d}} 14^{\mathrm{m}} 56^{\mathrm{s}} \cdot 9$ & $1.1_{-0.42}^{+0.52}$ & $48.55_{-17.9}^{+20.2}$ \\
\hline Mrk 1210 & $08^{\mathrm{h}} 04^{\mathrm{m}} 05^{\mathrm{s}} .86$ & $+05^{\mathrm{d}} 06^{\mathrm{m}} 49^{\mathrm{s}} .8$ & $3.2_{-0.61}^{+0.60}$ & $30.46_{-4.67}^{+4.67}$ \\
\hline MCG-01-22-006 & $08^{\mathrm{h}} 23^{\mathrm{m}} 01 \stackrel{\mathrm{s}}{.} 10$ & $-04^{\mathrm{d}} 56^{\mathrm{m}} 05^{\mathrm{s}} .5$ & $0.85_{-0.40}^{+0.45}$ & $49.38_{-16.8}^{+18.8}$ \\
\hline MCG+11-11-032 & $08^{\mathrm{h}} 55^{\mathrm{m}} 12^{\mathrm{s}} .54$ & $+64^{\mathrm{d}} 23^{\mathrm{m}} 45^{\mathrm{s}} \cdot 6$ & $1.3_{-0.27}^{+0.29}$ & $63.38_{-10.2}^{+13.1}$ \\
\hline Mrk 18 & $09^{\mathrm{h}} 01^{\mathrm{m}} 58.39$ & $+60^{\mathrm{d}} 09^{\mathrm{m}} 06^{\mathrm{s}} .2$ & $2.4_{-0.40}^{+0.20}$ & $78.11_{-32.2}^{+8.24}$ \\
\hline IC 2461 & $09^{\mathrm{h}} 19^{\mathrm{m}} 58^{\mathrm{s}} .03$ & $+37^{\mathrm{d}} 11^{\mathrm{m}} 28.5$ & $2.3_{-0.47}^{+0.29}$ & $70.80_{-9.63}^{+10.8}$ \\
\hline MCG-01-24-012 & $09^{\mathrm{h}} 20^{\mathrm{m}} 46^{\mathrm{s}} \cdot 25$ & $-08^{\mathrm{d}} 03^{\mathrm{m}} 22^{\mathrm{s}} \cdot 1$ & $2.3_{-0.46}^{+0.49}$ & $43.76_{-6.00}^{+9.25}$ \\
\hline 2MASXJ09235371-3141305 & $09^{\mathrm{h}} 23^{\mathrm{m}} 53^{\mathrm{s}} .73$ & $-31^{\mathrm{d}} 41^{\mathrm{m}} 30^{\mathrm{s}} .7$ & $1.6_{-0.43}^{+0.40}$ & $46.95_{-16.7}^{+19.8}$ \\
\hline NGC2992 & $09^{\mathrm{h}} 45^{\mathrm{m}} 42^{\mathrm{s}} .05$ & $-14^{\mathrm{d}} 19^{\mathrm{m}} 34^{\mathrm{s}} .9$ & $2.0_{-0.39}^{+0.49}$ & $47.76_{-18.2}^{+23.0}$ \\
\hline NGC3079 & $10^{\mathrm{h}} 01^{\mathrm{m}} 57^{\mathrm{s}} .80$ & $+55^{\mathrm{d}} 40^{\mathrm{m}} 47^{\mathrm{s}} \cdot 2$ & $1.1_{-0.41}^{+0.50}$ & $51.30_{-18.0}^{+18.1}$ \\
\hline ESO 263-G013 & $10^{\mathrm{h}} 09^{\mathrm{m}} 48^{\mathrm{s}} \cdot 21$ & $-42^{\mathrm{d}} 48^{\mathrm{m}} 40^{\mathrm{s}} \cdot 4$ & $1.7_{-0.40}^{+0.45}$ & $49.35_{-7.84}^{+11.8}$ \\
\hline NGC3281 & $10^{\mathrm{h}} 31^{\mathrm{m}} 52^{\mathrm{s}} .09$ & $-34^{\mathrm{d}} 51^{\mathrm{m}} 13.3$ & $2.1_{-0.59}^{+0.65}$ & $34.51_{-9.50}^{+12.6}$ \\
\hline MCG +12-10-067 & $10^{\mathrm{h}} 44^{\mathrm{m}} 08^{\mathrm{s}} \cdot 54$ & $+70^{\mathrm{d}} 24^{\mathrm{m}} 19^{\mathrm{s}} .3$ & $1.8_{-0.26}^{+0.35}$ & $59.41_{-10.7}^{+14.0}$ \\
\hline MCG+06-24-008 & $10^{\mathrm{h}} 44^{\mathrm{m}} 48^{\mathrm{s}} .97$ & $+38^{\mathrm{d}} 10^{\mathrm{m}} 51^{\mathrm{s}} \cdot 6$ & $1.1_{-0.45}^{+0.57}$ & $41.94_{-14.9}^{+19.8}$ \\
\hline UGC5881 & $10^{\mathrm{h}} 46^{\mathrm{m}} 42^{\mathrm{s}} \cdot 52$ & $+25^{\mathrm{d}} 55^{\mathrm{m}} 53^{\mathrm{s}} .6$ & $2.3_{-0.30}^{+0.43}$ & $60.28_{-12.7}^{+12.5}$ \\
\hline NGC3393 & $10^{\mathrm{h}} 48^{\mathrm{m}} 23^{\mathrm{s}} \cdot 46$ & $-25^{\mathrm{d}} 09^{\mathrm{m}} 43^{\mathrm{s}} .4$ & $2.1_{-0.38}^{+0.44}$ & $50.11_{-7.45}^{+12.0}$ \\
\hline Mrk 728 & $11^{\mathrm{h}} 01^{\mathrm{m}} 01^{\mathrm{s}} .78$ & $+11^{\mathrm{d}} 02^{\mathrm{m}} 48^{\mathrm{s}} .9$ & $1.7_{-0.29}^{+0.36}$ & $59.45_{-11.5}^{+14.5}$ \\
\hline 2MASXJ11364205-6003070 & $11^{\mathrm{h}} 36^{\mathrm{m}} 42^{\mathrm{s}} .05$ & $-60^{\mathrm{d}} 03^{\mathrm{m}} 06^{\mathrm{s}} .7$ & $1.9_{-0.40}^{+0.47}$ & $47.47_{-15.6}^{+17.7}$ \\
\hline NGC3786 & $11^{\mathrm{h}} 39^{\mathrm{m}} 42^{\mathrm{s}} .55$ & $+31^{\mathrm{d}} 54^{\mathrm{m}} 333^{\mathrm{s}} .4$ & $1.1_{-0.43}^{+0.48}$ & $47.98_{-16.6}^{+18.0}$ \\
\hline NGC4388 & $12^{\mathrm{h}} 25^{\mathrm{m}} 46^{\mathrm{s}} .75$ & $+12^{\mathrm{d}} 39^{\mathrm{m}} 43^{\mathrm{s}} \cdot 5$ & $2.1_{-0.46}^{+0.58}$ & $43.44_{-12.1}^{+15.2}$ \\
\hline LEDA170194 & $12^{\mathrm{h}} 39^{\mathrm{m}} 06^{\mathrm{s}} .28$ & $-16^{\mathrm{d}} 10^{\mathrm{m}} 47^{\mathrm{s}} .1$ & $1.6_{-0.31}^{+0.37}$ & $56.67_{-13.4}^{+15.8}$ \\
\hline NGC4941 & $13^{\mathrm{h}} 04^{\mathrm{m}} 13^{\mathrm{s}} .14$ & $-05^{\mathrm{d}} 33^{\mathrm{m}} 05^{\mathrm{s}} .8$ & $1.5_{-0.24}^{+0.29}$ & $65.13_{-9.78}^{+12.7}$ \\
\hline NGC4992 & $13^{\mathrm{h}} 09^{\mathrm{m}} 05^{\mathrm{s}} \cdot 60$ & $+11^{\mathrm{d}} 38^{\mathrm{m}} 03^{\mathrm{s}} \cdot 0$ & $1.3_{-0.31}^{+0.40}$ & $54.30_{-9.12}^{+12.4}$ \\
\hline Mrk 248 & $13^{\mathrm{h}} 15^{\mathrm{m}} 17^{\mathrm{s}} .27$ & $+44^{\mathrm{d}} 24^{\mathrm{m}} 25^{\mathrm{s}} .6$ & $2.0_{-0.46}^{+0.71}$ & $41.76_{-14.7}^{+32.7}$ \\
\hline ESO 509-IG066 & $13^{\mathrm{h}} 34^{\mathrm{m}} 40^{\mathrm{s}} \cdot 40$ & $-23^{\mathrm{d}} 26^{\mathrm{m}} 46^{\mathrm{s}} .0$ & $2.8_{-0.64}^{+0.65}$ & $32.25_{-7.53}^{+9.56}$ \\
\hline NGC5252 & $13^{\mathrm{h}} 38^{\mathrm{m}} 15^{\mathrm{s}} .96$ & $+04^{\mathrm{d}} 32^{\mathrm{m}} 33^{\mathrm{s}} \cdot 3$ & $0.91_{-0.41}^{+0.41}$ & $55.65_{-18.5}^{+17.8}$ \\
\hline NGC5273 & $13^{\mathrm{h}} 42^{\mathrm{m}} 08^{\mathrm{s}} \cdot 34$ & $+35^{\mathrm{d}} 39^{\mathrm{m}} 15^{\mathrm{s}} \cdot 2$ & $1.3_{-0.43}^{+0.47}$ & $53.76_{-18.4}^{+17.1}$ \\
\hline NGC5674 & $14^{\mathrm{h}} 33^{\mathrm{m}} 52^{\mathrm{s}} \cdot 24$ & $+05^{\mathrm{d}} 27^{\mathrm{m}} 29 \stackrel{\mathrm{s}}{6}$ & $0.44_{-0.39}^{+0.53}$ & $48.19_{-18.9}^{+19.6}$ \\
\hline NGC5728 & $14^{\mathrm{h}} 42^{\mathrm{m}} 23^{\mathrm{s}} .90$ & $-17^{\mathrm{d}} 15^{\mathrm{m}} 11^{\mathrm{s}} .1$ & $2.0_{-0.45}^{+0.39}$ & $63.18_{-11.3}^{+11.5}$ \\
\hline IC $4518 \mathrm{~A}$ & $14^{\mathrm{h}} 57^{\mathrm{m}} 41^{\mathrm{s}} .18$ & $-43^{\mathrm{d}} 07^{\mathrm{m}} 55^{\mathrm{s}} \cdot 6$ & $2.6_{-0.43}^{+0.59}$ & $45.09_{-15.6}^{+17.2}$ \\
\hline 2MASXJ15064412+0351444 & $15^{\mathrm{h}} 06^{\mathrm{m}} 44^{\mathrm{s}} .13$ & $+03^{\mathrm{d}} 51^{\mathrm{m}} 44^{\mathrm{s}} .4$ & $1.6_{-0.50}^{+0.50}$ & $51.64_{-17.2}^{+18.1}$ \\
\hline NGC5899 & $15^{\mathrm{h}} 15^{\mathrm{m}} 03^{\mathrm{s}} \cdot 22$ & $+42^{\mathrm{d}} 02^{\mathrm{m}} 59^{\mathrm{s}} .5$ & $1.2_{-0.44}^{+0.47}$ & $63.82_{-17.4}^{+14.9}$ \\
\hline MCG+11-19-006 & $15^{\mathrm{h}} 19^{\mathrm{m}} 33^{\mathrm{s}} .69$ & $+65^{\mathrm{d}} 35^{\mathrm{m}} 58.5$ & $1.8_{-0.56}^{+0.76}$ & $34.46_{-12.0}^{+14.6}$ \\
\hline MCG-01-40-001 & $15^{\mathrm{h}} 33^{\mathrm{m}} 20^{\mathrm{s}} .71$ & $-08^{\mathrm{d}} 42^{\mathrm{m}} 01^{\mathrm{s}} .9$ & $2.8_{-0.29}^{+0.42}$ & $54.32_{-8.39}^{+11.6}$ \\
\hline NGC5995 & $15^{\mathrm{h}} 48^{\mathrm{m}} 24.96$ & $-13^{\mathrm{d}} 45^{\mathrm{m}} 27^{\mathrm{s}} .9$ & $1.6_{-0.34}^{+0.45}$ & $50.36_{-12.6}^{+16.3}$ \\
\hline MCG+14-08-004 & $16^{\mathrm{h}} 19^{\mathrm{m}} 19^{\mathrm{s}} \cdot 26$ & $+81^{\mathrm{d}} 02^{\mathrm{m}} 48^{\mathrm{s}} \cdot 6$ & $1.6_{-0.45}^{+0.55}$ & $42.77_{-13.1}^{+12.1}$ \\
\hline
\end{tabular}


Table 1

(Continued)

\begin{tabular}{|c|c|c|c|c|}
\hline \multirow[b]{2}{*}{ Name } & \multirow[b]{2}{*}{$\begin{array}{l}\text { R.A. } \\
(\text { J2000) }\end{array}$} & \multirow[b]{2}{*}{$\begin{array}{l}\text { Decl. } \\
(\text { J2000) }\end{array}$} & \multicolumn{2}{|c|}{ IR Parameters } \\
\hline & & & $\begin{array}{l}\text { AGN Slope } \\
\quad(\alpha)\end{array}$ & $\begin{array}{c}\text { Turnover } \\
\text { Wavelength }\left(\lambda_{\mathrm{C}}\right)\end{array}$ \\
\hline NGC6240 & $16^{\mathrm{h}} 52^{\mathrm{m}} 58^{\mathrm{s}} .87$ & $+02^{\mathrm{d}} 24^{\mathrm{m}} 03^{\mathrm{s}} .3$ & $2.8_{-0.44}^{+0.87}$ & $40.29_{-12.9}^{+34.8}$ \\
\hline NGC6300 & $17^{\mathrm{h}} 16^{\mathrm{m}} 59^{\mathrm{s}} \cdot 47$ & $-62^{\mathrm{d}} 49^{\mathrm{m}} 14^{\mathrm{s}} \cdot 0$ & $1.7_{-0.32}^{+0.30}$ & $65.46_{-10.3}^{+12.6}$ \\
\hline MCG $+07-37-031$ & $18^{\mathrm{h}} 16^{\mathrm{m}} 11^{\mathrm{s}} .55$ & $+42^{\mathrm{d}} 39^{\mathrm{m}} 37^{\mathrm{s}} .2$ & $2.3_{-0.35}^{+0.51}$ & $47.79_{-14.8}^{+21.5}$ \\
\hline IC 4709 & $18^{\mathrm{h}} 24^{\mathrm{m}} 19^{\mathrm{s}} .39$ & $-56^{\mathrm{d}} 22^{\mathrm{m}} 09^{\mathrm{s}} \cdot 0$ & $1.8_{-0.32}^{+0.40}$ & $52.87_{-8.34}^{+11.4}$ \\
\hline ESO 103-G035 & $18^{\mathrm{h}} 38^{\mathrm{m}} 20^{\mathrm{s}} \cdot 34$ & $-65^{\mathrm{d}} 25^{\mathrm{m}} 39^{\mathrm{s}} \cdot 2$ & $2.9_{-0.69}^{+0.69}$ & $29.96_{-5.67}^{+5.93}$ \\
\hline 2MASXJ20183871+4041003 & $20^{\mathrm{h}} 18^{\mathrm{m}} 38^{\mathrm{s}} .72$ & $+40^{\mathrm{d}} 41^{\mathrm{m}} 00^{\mathrm{s}} \cdot 2$ & $0.93_{-0.42}^{+0.56}$ & $44.93_{-17.3}^{+19.1}$ \\
\hline $\mathrm{MCG}+04-48-002$ & $20^{\mathrm{h}} 28^{\mathrm{m}} 35^{\mathrm{s}} .06$ & $+25^{\mathrm{d}} 44^{\mathrm{m}} 00^{\mathrm{s}} .0$ & $1.3_{-0.43}^{+0.53}$ & $47.11_{-17.9}^{+18.7}$ \\
\hline IC 5063 & $20^{\mathrm{h}} 52^{\mathrm{m}} 02^{\mathrm{s}} .34$ & $-57^{\mathrm{d}} 04^{\mathrm{m}} 07^{\mathrm{s}} .6$ & $2.2_{-0.48}^{+0.47}$ & $43.60_{-5.44}^{+9.12}$ \\
\hline $\mathrm{MCG}+06-49-019$ & $22^{\mathrm{h}} 27^{\mathrm{m}} 05^{\mathrm{s}} \cdot 78$ & $+36^{\mathrm{d}} 21^{\mathrm{m}} 41^{\mathrm{s}} .7$ & $1.5_{-0.29}^{+0.34}$ & $60.78_{-10.8}^{+13.1}$ \\
\hline $\mathrm{MCG}+01-57-016$ & $22^{\mathrm{h}} 40^{\mathrm{m}} 17^{\mathrm{s}} .05$ & $+08^{\mathrm{d}} 03^{\mathrm{m}} 14^{\mathrm{s}} .1$ & $1.9_{-0.33}^{+0.37}$ & $54.71_{-12.2}^{+13.4}$ \\
\hline NGC7582 & $23^{\mathrm{h}} 18^{\mathrm{m}} 23^{\mathrm{s}} \cdot 50$ & $-42^{\mathrm{d}} 22^{\mathrm{m}} 14^{\mathrm{s}} .0$ & $2.1_{-0.43}^{+0.59}$ & $43.57_{-14.9}^{+20.5}$ \\
\hline 2MASXJ23303771+7122464 & $23^{\mathrm{h}} 30^{\mathrm{m}} 37^{\mathrm{s}} .69$ & $+71^{\mathrm{d}} 22^{\mathrm{m}} 46^{\mathrm{s}} .5$ & $1.6_{-0.45}^{+0.63}$ & $42.37_{-16.9}^{+19.9}$ \\
\hline PKS 2331-240 & $23^{\mathrm{h}} 33^{\mathrm{m}} 55^{\mathrm{s}} \cdot 24$ & $-23^{\mathrm{d}} 43^{\mathrm{m}} 40^{\mathrm{s}} \cdot 66$ & $1.2_{-0.11}^{+0.12}$ & $137.7_{-10.5}^{+9.34}$ \\
\hline
\end{tabular}

Note. Names (Column 1) and coordinates (Columns 2 and 3) of our sample, along with two parameters from the SED decomposition from S17: the slope of the exponentially cutoff power law (Column 4) and its turnover wavelength (Column 5). Further details of the modeling are given in Section 3.2.

beamed sources) and of the Swift/BAT Sy2 AGN sample. As shown in Figure 1, we compared the distributions of the 14-195 keV Swift/BAT luminosities from the 70 month catalog (Baumgartner et al. 2013) for all unbeamed BAT AGNs at $z<0.05$ (gray), the full S17 sample (yellow), the $z<0.05$ B 18 subsample (green), and our overlap sample of 69 (blue). Table 2 contains the results of Kolmogorov-Smirnov $(\mathrm{KS})^{23}$ tests on these distributions, as well as the comparison of the full Sy2 subset of the $z<0.05$ BAT AGN sample. In each comparison, we cannot reject the null hypothesis that the two samples are consistent with originating from the same population. As a result, we consider our sample to be representative of the complete Swift/BAT AGN sample at redshifts $z<0.05$.

\section{Observations and Data Processing}

\subsection{NUSTAR and X-Ray Spectral Analysis}

Detailed discussion of the NUSTAR analysis can be found in B18. We briefly summarize it here. The reduced spectra were binned to have constant signal-to-noise ratios in each energy bin. Each spectrum is fit in the full NUSTAR energy band $(3-79 \mathrm{keV})$ in combination with the Swift/XRT data $(0.2-10 \mathrm{keV})$ with XSPEC (Arnaud 1996). The model used ${ }^{24}$ is composed of several components behind an obscuration screen due to foreground absorption by the Milky Way: (1) an absorbed, exponentially cutoff power law for the underlying intrinsic emission; (2) an unabsorbed exponentially cutoff power law to account for the soft emission that may be due to optically thin scattering, X-ray binaries, and/or other ionized emission within the galaxy; and (3) a reflection component using just the reflection part of the pexrav (Magdziarz \& Zdziarski 1995) model combined with an unresolved $\left(\sigma=10^{-3} \mathrm{keV}\right)$ Gaussian $\mathrm{Fe} \mathrm{K} \alpha$ line at a fixed rest frame

\footnotetext{
${ }^{23}$ Using the IDL routine KSTWO.

${ }^{24}$ const $\times$ phabs $\times$ (zphabs $\times$ cabs $\times$ cutoffpl + const $\times$ cutoffpl + pexrav + zgauss).
}

energy of $6.4 \mathrm{keV}$. The unabsorbed power law is primarily constrained by the Swift/XRT data, which is not sufficient to independently constrain the slope, so it is assumed to be the same as that of the intrinsic power law. High energy cutoffs are fixed at $300 \mathrm{keV}$, which was justified post facto (B18).

In the pexrav model, the reflection parameter is restricted to be below zero (i.e., the range in which only the reflection component appears), and a solar metallicity and an inclination of the default $60^{\circ}$ are assumed. ${ }^{25}$ Although the pexrav model is less physically motivated than some more complex models for reflection (see, for example, Gandhi et al. 2014; Annuar et al. 2015, and Baloković et al. 2014, 2018 for comparisons between such models and pexrav-based modeling), it has the benefit of capturing the general nature of the reflection with the fewest possible parameters. A detailed systematic comparison of pexrav and geometrically motivated torus models for a large sample of 120 AGNs, including those used in this work, is in preparation (Baloković et al. 2018, in preparation), with some preliminary results outlined in Baloković (2017).

The resulting fits yield the following parameters: the obscuration column density $N_{\mathrm{H}}$, the power-law slope $\Gamma$, the equivalent width of the $\mathrm{Fe} \mathrm{K} \alpha$ line $\left(\mathrm{EW}_{\mathrm{Fe} \mathrm{K} \alpha}\right)$, the relative normalization of the unabsorbed power law, and the reflection parameter from the pexrav model $\left(R_{\mathrm{pex}}=|R|\right.$, where $R$ is the negative number from the XSPEC fitting). These parameters are given in Table 3. In addition to the luminosities described below, we primarily use the reflection parameter and the column density in the analysis that follows, although Appendix A contains additional discussion of the other X-ray parameters.

For three AGNs, the quality of the spectra was insufficient to robustly fit all the parameters, so we fixed one of the parameters: for ESO 005-G004, we fixed the power-law slope

\footnotetext{
25 There is a degeneracy in pexrav between inclination and the normalization of the reflection component (e.g., Figure 1 in Dauser et al. 2016). However, changes in the inclination have very little effect on the shape of the spectrum. We fix the inclination to handle the normalization only through the reflection parameter $R_{\text {pex }}$.
} 
Table 2

Sample Comparisons

\begin{tabular}{|c|c|c|c|c|}
\hline & Samples & & KS Statistic & KS Probability \\
\hline$z<0.05$ BAT AGN (gray) & versus & S17 (Herschel; yellow) & 0.044 & $90.5 \%$ \\
\hline$z<0.05$ BAT AGN (gray) & versus & $z<0.05$ B $18(N u S T A R ;$ green $)$ & 0.109 & $32.1 \%$ \\
\hline$z<0.05$ BAT AGN (gray) & versus & this paper (blue) & 0.103 & $56.0 \%$ \\
\hline S17 (Herschel; yellow) & versus & this paper (blue) & 0.130 & $29.0 \%$ \\
\hline$z<0.05$ B $18(N u S T A R ;$ green $)$ & versus & this paper (blue) & 0.055 & $99.96 \%$ \\
\hline$z<0.05$ BAT Sy 2 AGN $^{\mathrm{a}}$ & versus & this paper (blue) & 0.080 & $90.6 \%$ \\
\hline
\end{tabular}

Notes. Results of performing Kolmogorov-Smirnov (KS) tests on the distributions shown in Figure 1. For ease of comparison to the figure, we note the associated color of the distribution in Columns 1 and 2. The BAT AGN samples are selected from the 70 month catalog. Column 3 has the KS statistic, corresponding to the largest separation between the cumulative distribution functions of the two samples. Column 4 has the associated probability of the null hypothesis that the two samples originate from the same parent population. We require a probability less than $0.3 \%$ to reject the null hypothesis at a $3 \sigma$ confidence level.

${ }^{a}$ This subset is not explicitly shown in Figure 1.

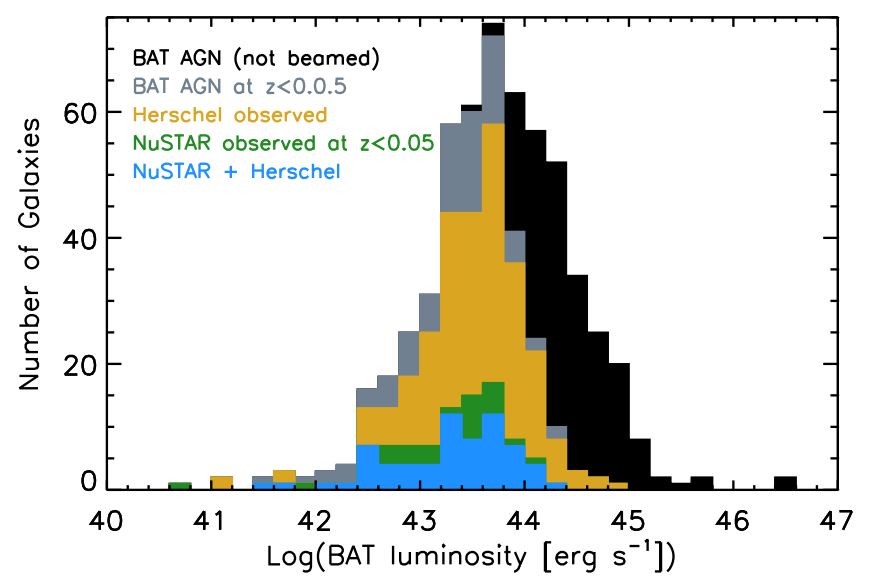

Figure 1. Histogram of the $14-195 \mathrm{keV}$ Swift/BAT luminosity of all BAT AGN (black) from the 70-month Swift/BAT catalog with the exclusion of beamed sources, as well as its subset after a redshift cut at $z=0.05$ (gray) compared to the samples observed with Herschel (yellow; S17) and NuSTAR (green; B18 with a $z=0.05$ redshift cut). The overlap sample that we use is shown in blue. Within the redshift range of $z<0.05$, the NUSTAR and Herschel samples are statistically representative of the BAT AGN, as is our joint sample (see Table 2).

at the typical AGN slope of 1.8 (Piconcelli et al. 2005; Dadina 2008); for MCG+04-48-002, $\Gamma=1.8$ produced an unstable fit, so we used a fixed $\Gamma=1.7$ instead; for the Compton-thick source LEDA96373, the simple model fit only stabilized if the column was fixed, so we used a $\log \left(N_{\mathrm{H}}\left[\mathrm{cm}^{-2}\right]\right)=24.1$, which has been confirmed as reasonable using more complex models (see B18 for further details). For one determination of $N_{\mathrm{H}}$, seven of Fe $\mathrm{K} \alpha$ and nine of the reflection parameter, there is a best fitted value but the lower limit on its uncertainty is poorly constrained.

For our analysis, we use both the spectral parameters described above and the observed, reflected, and intrinsic $10-50 \mathrm{keV}$ luminosities. The intrinsic luminosity is corrected for both absorption (which decreases observed flux) and reflection (which increases observed flux) and is, therefore, smaller than the unabsorbed luminosity, which is only corrected for obscuration. These are given in Table 4. The reflected luminosity is given by the reflection parameter times the intrinsic luminosity.

We chose to use the $10-50 \mathrm{keV}$ luminosity for this analysis, but we also tested the analysis we undertook using the intrinsic $2-10 \mathrm{keV}$ luminosity. We found very similar results, because the intrinsic luminosities in both bands are calculated using the same power-law model, in which the photon index ${ }^{26}, \Gamma$, relates to flux density with $F_{\nu} \propto \nu^{-\Gamma+1}$. The range of $\Gamma$ in our sample introduces $\sim 0.1$ dex of scatter in the intrinsic X-ray luminosity; however, this is relatively small compared to the 0.4 dex scatter in the ratio between IR and X-ray emission, as we discuss further below.

\subsection{Herschel and Infrared Spectral Energy Distribution Fitting}

Meléndez et al. (2014) and Shimizu et al. (2016) describe in detail the Herschel observations of 313 Swift/BAT galaxies taken by the Photodetector Array Camera and Spectrometer (PACS; Poglitsch et al. 2010) and Spectral and Photometric Imaging Receiver (SPIRE; Griffin et al. 2010), respectively, as well as their reduction and analysis. PACS observations were taken at $70 \mu \mathrm{m}$ and $160 \mu \mathrm{m}$, whereas SPIRE observations were taken at $250 \mu \mathrm{m}, 350 \mu \mathrm{m}$, and $500 \mu \mathrm{m}$, all primarily as part of a Cycle 1 program (OT1_rmushot_1; PI R. Mushotzky). We briefly summarize the SED analysis done with them below.

S17 combined these data with archival Wide-field Infrared Survey Explorer (WISE; Wright et al. 2010) photometry from 3.4 to $22 \mu \mathrm{m}$ to create and fit SEDs and determine the relative contributions of the AGN and star formation (SF) to the IR SED. They model the SEDs as the combination of a modified blackbody (where the dust emissivity inversely depends on wavelength to the power $\beta=2$ ) and an exponentially cutoff power law (with a fitted power $\alpha$ ) with turnover wavelength $\left(\lambda_{C}\right)$. The fitting is done within a Bayesian framework with a Markov chain Monte Carlo to determine the posterior probability distribution functions of the parameters. Through identical analysis of the Herschel Reference Survey (HRS; Boselli et al. 2010), a sample of galaxies that contain only low-luminosity AGNs if any, S17 showed that a component of the power-law emission was due to SF. They used this HRS analysis to determine the correction needed according to the luminosity of the modified blackbody component, which is strictly due to SF.

As a means of testing this SED decomposition, we crosscorrelated our sample with that of Asmus et al. (2014), who performed high-spatial-resolution MIR photometry of local AGNs. Our samples have 26 AGNs in common. Figure 2 shows that the $12 \mu \mathrm{m}$ luminosities from Asmus et al. (2014) correlate very well with the SED-derived AGN $(8-1000 \mu \mathrm{m})$ IR luminosities, with scatter about a factor of 3 lower than that of the IR luminosities before the decomposition. The comparison line shown assumes

\footnotetext{
$\overline{{ }^{26} \text { Defined such that } P_{E}\left[\text { photons } \mathrm{s}^{-1} \mathrm{keV}^{-1}\right]} \propto E^{-\Gamma}$.
} 


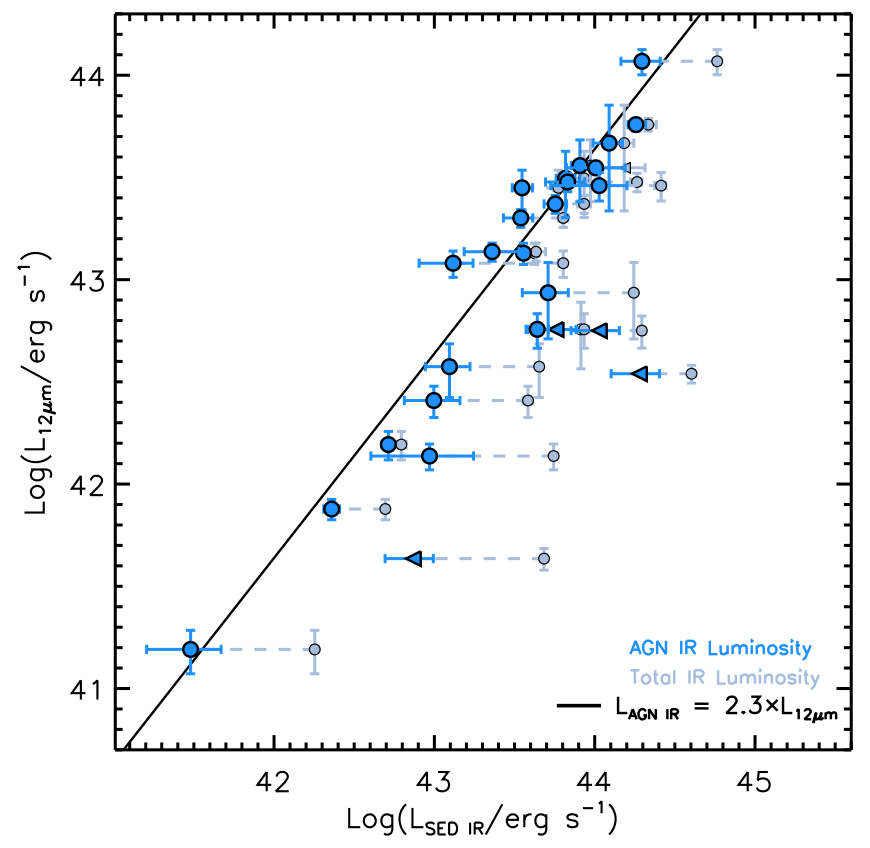

Figure 2. AGN IR $(8-1000 \mu \mathrm{m})$ luminosity in blue from the SED decomposition compared to resolved nuclear $12 \mu \mathrm{m}$ luminosities from Asmus et al. (2014) where available. We also show the total IR luminosity before the decomposition (gray) and the expected relation (solid line) between the 8-1000 $\mu \mathrm{m}$ luminosity and the $12 \mu$ m luminosity from the AGN SED models of Mullaney et al. (2011). Our AGN IR luminosities typically agree within their uncertainties with this expected relation, demonstrating the reliability of the SED decomposition compared to high-spatialresolution MIR observations. Triangles indicate $3 \sigma$ upper limits in a direction of the point.

the ratio between $12 \mu \mathrm{m}$ and the broadband AGN IR luminosities from Mullaney et al. (2011). The relation between the AGN IR and resolved $12 \mu \mathrm{m}$ luminosities of our sample typically agrees with this ratio within the uncertainties of the measurements.

For our analysis, we primarily used the derived AGN IR luminosity. We also examined the SF IR luminosity, total IR luminosity $(8-1000 \mu \mathrm{m})$, the AGN luminosity fraction, and the two parameters from the power-law (AGN) component ( $\alpha$ and $\lambda_{\mathrm{C}}$ ). These parameters are given in Tables 1 and 4. For four AGNs, the AGN IR luminosity is a lower limit, likewise restricted by an upper limit on the total IR luminosity. For our analysis, we assign these AGNs the average luminosity between these limits using the range to the limits as the uncertainty on these values.

\section{Analysis and Discussion}

We explored the relations between and within the IR and X-ray properties, including (1) NUSTAR spectral parameters; (2) IR modeling parameters; (3) intrinsic, reflected, and observed X-ray luminosities in the $10-50 \mathrm{keV}$ band; (4) AGN, total, and SF IR luminosities; and (5) ratios of an IR luminosity to an X-ray luminosity. We show a subset in Figures 3 and 7. For each of these pairings, we use the ASURV survival analysis package to calculate the Spearman $\rho$ rank correlation (Isobe et al. 1986; Lavalley et al. 1992), thereby taking the limits into account. This statistic tests the null hypothesis that there is no monotonic relation between the parameters. We define a significant correlation as one that rejects this hypothesis by having a probability less than $3 \times 10^{-3}(\log [p] \leqslant-2.52)$, corresponding to approximately $3 \sigma$. To calculate the confidence interval of the Spearman statistic and associated probability, we undertook a bootstrap analysis in which we pick 1000 samples and ran the ASURV analysis on each. ${ }^{27}$

In the sections below, we discuss in detail how the correlation between reflected X-ray and IR emission implies a common source of reprocessing of the intrinsic emission and the implications of the relationship between the reflection parameter and the ratio of IR-to-X-ray emission for the distribution of covering fractions for all AGNs. Appendix A contains additional discussion of the relations of other X-ray and IR parameters.

\subsection{Relationship Between Infrared and X-Ray Intrinsic and Reflected Luminosities}

We begin by comparing X-ray intrinsic and reflected luminosities to the IR luminosity of the AGN. Correlations between intrinsic X-ray and IR luminosities have long been known, and we show four X-ray to MIR literature relations in Figure 3(a) (Asmus et al. 2015, solid blue; Chen et al. 2017, yellow dash-triple dotted; Gandhi et al. 2009, red long-dashed; and Fiore et al. 2009, green dotted), adapted to account for different IR and X-ray bands. Specifically, the Fiore and Chen relations were derived for IR luminosity at $6 \mu \mathrm{m}$, whereas the Gandhi and Asmus relations are calculated at $12 \mu \mathrm{m}$. We convert the relations to the $8-1000 \mu \mathrm{m}$ IR luminosity measured by $\mathrm{S} 17$ using the typical ratios provided by Mullaney et al. (2011). Similarly, the four relations are derived for X-ray luminosities in the $2-10 \mathrm{keV}$ band. We convert to the 10-50 keV band, assuming a power law with $\Gamma=1.8$ (Netzer 2015; consistent with our median $\Gamma$ ), resulting in a multiplicative factor of 1.38. On these relations, we overlay the AGN's IR luminosity (from S17) against the intrinsic $10-50 \mathrm{keV}$ luminosity from the fits by B18.

We find a correlation between these luminosities (Figure $3(\mathrm{a}) ; \quad \rho=0.47 \pm 0.10 ; \quad \log (p)=-4.03 \pm 1.35){ }^{28}$ The correlation is less significant but still very suggestive when we use fluxes (Figure 3(c); $\rho=0.34 \pm 0.11 ; \log$ $(p)=-2.28 \pm 1.17)$ instead of luminosities, which confirms that the correlations are not purely due to those that can be introduced into luminosity correlations by the effects of distance (e.g., Feigelson \& Berg 1983).

We also find a significant correlation between the reflected X-ray and IR luminosities $(\rho=0.61 \pm 0.08 ; \log (p)=$ $-6.29 \pm 1.51$ for luminosities in Figure 3(b); $\rho=0.49 \pm$ $0.11 ; \log (p)=-4.22 \pm 1.58$ for fluxes in Figure $3(\mathrm{~d}))$. On the basis of these confidence intervals, we find a suggestive difference in the correlations, present in both the luminosities and the fluxes, of $\sim 1 \sigma$, corresponding to a confidence level of $70 \%$. Because the size of our confidence intervals is primarily driven by our sample size (see footnote 28), a larger sample will be needed to conclusively determine whether the reflected emission is indeed significantly more correlated than the intrinsic emission.

\footnotetext{
27 The code we wrote to do this analysis is available at https://github.com/ lalanz/bootstrap_asurv.

${ }^{28}$ We used bootstrap samples picked with replacement from our data. We found that this methodology yielded a larger confidence interval than selecting samples using Gaussian distributions centered at each detection with widths given by their uncertainties. This difference indicates that the uncertainty in our correlations is primarily driven by the sample size and/or intrinsic scatter. We report the median and confidence interval of the statistic and corresponding probability.
} 

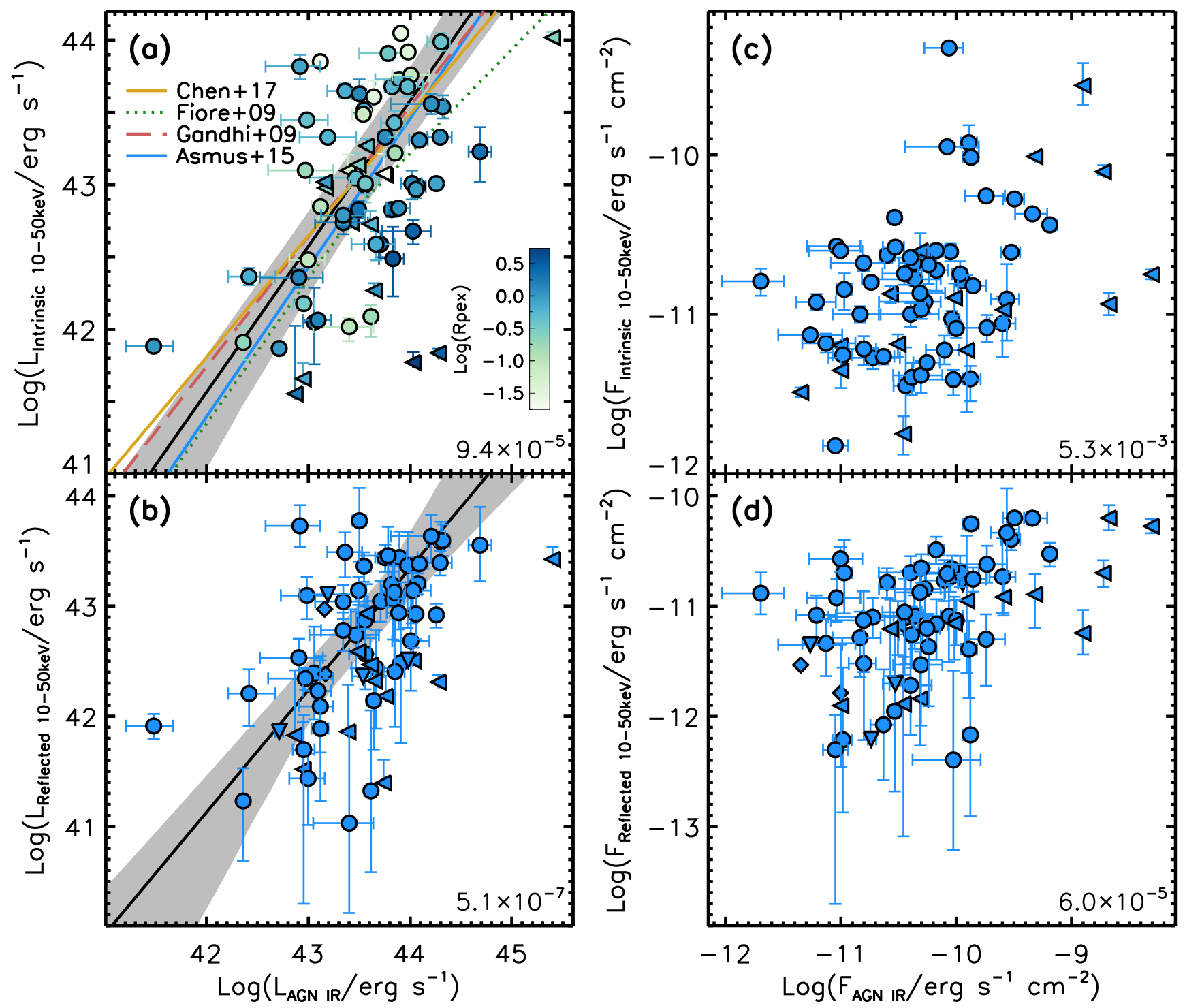

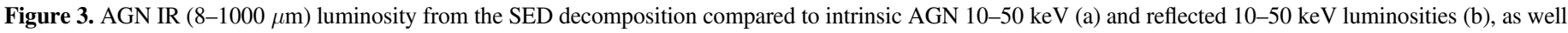

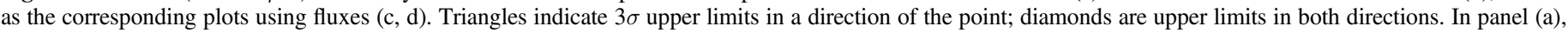

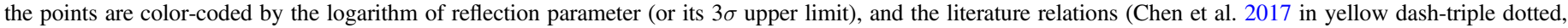

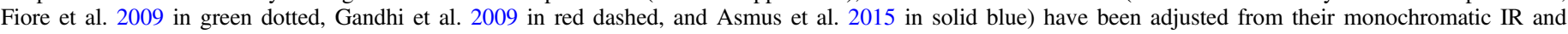

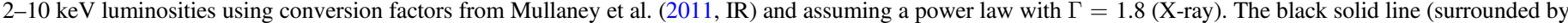

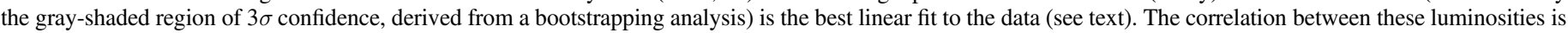
also seen between the fluxes; the probability of not having a correlation is given in the lower right.

We also test this relative correlation using a comparative partial correlation test. ${ }^{29}$ We calculate the correlation of the reflected X-ray luminosity with the residual after the correlation between the intrinsic X-ray luminosity with the IR luminosity has been removed, as well as the correlation when we reverse the roles of the reflected and intrinsic X-ray luminosities. We find that the partial correlation is stronger with the reflected $\mathrm{X}$-ray luminosity $\left(p_{\mathrm{X}_{\mathrm{R}} I R \cdot \mathrm{X}_{\mathrm{I}}}=0.36\right)$ than with the intrinsic $\mathrm{X}$-ray luminosity $\left(p_{\mathrm{X}_{\mathrm{I}} \mathrm{IR} \cdot \mathrm{X}_{\mathrm{R}}}=0.22\right)$. This difference remains when we uses fluxes instead of luminosities $\left(p_{\mathrm{X}_{\mathrm{R}} \mathrm{IR} \cdot \mathrm{X}_{\mathrm{I}}}=0.29\right.$ versus $p_{\mathrm{X}_{\mathrm{I}} \mathrm{IR} \cdot \mathrm{X}_{\mathrm{R}}}=0.18$ ).

To investigate the relations between these luminosities, we fit a line in Figures 3(a) and (b). To take into account

\footnotetext{
$\overline{{ }^{29}} \mathrm{We}$ used the IDL routine p_correlate solely with the detected luminosities.
}

uncertainties in both luminosities when fitting each line, we perform a fit using orthogonal regression, maximizing the likelihoods provided in Pihajoki (2017) for both uncensored and censored $^{30}$ data. We use the IDL package MPFIT's Levenberg-Marquardt algorithm to minimize the inverse of the likelihoods (More 1978; Markwardt 2009). We undertook a bootstrapping analysis using 10,000 samples selected with replacements in order to estimate the uncertainties in the slope and intercept. ${ }^{31}$

The black solid lines in Figures 3(a) and (b) show the results with the gray regions showing the $3 \sigma$ confidence range from the bootstrapping analysis. The relation with intrinsic X-ray luminosity has a slope of $1.01 \pm 0.10$, whereas the relation with the reflected

\footnotetext{
${ }^{30}$ We exclude points that are simultaneously censored in both luminosities.

31 The code we wrote to do the orthogonal fit and estimate its confidence interval is available at https://github.com/lalanz/orthogonal_regression.
} 
Table 3

NuSTAR Parameters

\begin{tabular}{|c|c|c|c|c|c|}
\hline \multirow[b]{2}{*}{ Name } & \multicolumn{5}{|c|}{ NuSTAR parameters } \\
\hline & $\begin{array}{r}\log \left(\mathrm{N}_{\mathrm{H}}\right) \\
\left(\mathrm{cm}^{-2}\right)\end{array}$ & Gamma & $\begin{array}{l}\mathrm{EW}(\mathrm{Fe} \mathrm{K} \alpha) \\
\quad(\mathrm{keV})\end{array}$ & $\begin{array}{l}\text { Reflection } \\
\text { Parameter }\end{array}$ & $\begin{array}{c}\text { Unabs. PL } \\
\text { Normalization }\end{array}$ \\
\hline LEDA136991 & $23.95_{-0.18}^{+0.17}$ & $1.711_{-0.127}^{+0.138}$ & $\left(7.50_{-1.96}^{+6.00}\right) \times 10^{-1}$ & $2.21_{-1.11}^{+2.69}$ & $<9.80 \times 10^{-1}$ \\
\hline NGC262 & $22.97_{-0.01}^{+0.01}$ & $1.747_{-0.019}^{+0.020}$ & $\left(4.47_{-1.18}^{+1.17}\right) \times 10^{-2}$ & $\left(5.79_{-0.75}^{+0.78}\right) \times 10^{-1}$ & $\left(2.06_{-0.91}^{+0.92}\right) \times 10^{-1}$ \\
\hline ESO 195-IG021 & $22.62_{-0.05}^{+0.04}$ & $1.876_{-0.073}^{+0.078}$ & $\left(1.35_{-0.36}^{+0.35}\right) \times 10^{-1}$ & $\left(5.15_{-2.86}^{+3.63}\right) \times 10^{-1}$ & $\left(4.83_{-3.65}^{+3.71}\right) \times 10^{-1}$ \\
\hline IC 1663 & $23.44_{-0.11}^{+0.09}$ & $1.571_{-0.168}^{+0.245}$ & $\left(4.04_{l}^{+14.6}\right) \times 10^{-2}$ & $\left(1.72_{l}^{+7.41}\right) \times 10^{-1}$ & $1.62_{-1.00}^{+2.18}$ \\
\hline NGC513 & $22.85_{-0.08}^{+0.08}$ & $1.699_{-0.111}^{+0.117}$ & $\left(1.75_{-0.63}^{+0.62}\right) \times 10^{-1}$ & $1.03_{-0.46}^{+0.64}$ & $2.49_{-0.92}^{+1.16}$ \\
\hline MCG-01-05-047 & $23.22_{-0.07}^{+0.06}$ & $1.807_{-0.101}^{+0.097}$ & $\left(3.38_{-0.84}^{+0.88}\right) \times 10^{-1}$ & $2.86_{-0.90}^{+1.27}$ & $1.32_{-0.56}^{+0.68}$ \\
\hline NGC788 & $23.89_{-0.03}^{+0.04}$ & $1.770_{-0.046}^{+0.047}$ & $\left(2.92_{-0.72}^{+0.77}\right) \times 10^{-1}$ & $\left(7.45_{-1.84}^{+2.34}\right) \times 10^{-1}$ & $1.25_{-0.36}^{+0.46}$ \\
\hline NGC1052 & $23.04_{-0.05}^{+0.04}$ & $1.516_{-0.040}^{+0.042}$ & $\left(1.63_{-0.43}^{+0.43}\right) \times 10^{-1}$ & $<3.30 \times 10^{-1}$ & $8.12_{-1.29}^{+1.53}$ \\
\hline 2MFGC 2280 & $24.20_{-0.06}^{+0.07}$ & $1.564_{-0.154}^{+0.161}$ & $\left(1.28_{-0.84}^{+1.03}\right) \times 10^{0}$ & $\left(5.78_{l}^{+9.12}\right) \times 10^{-2}$ & $\left(2.21_{-1.32}^{+2.34}\right) \times 10^{-1}$ \\
\hline NGC1365 & $23.30_{-0.02}^{+0.02}$ & $1.903_{-0.033}^{+0.034}$ & $\left(8.08_{-1.88}^{+1.87}\right) \times 10^{-2}$ & $2.98_{-0.36}^{+0.41}$ & $4.13_{-0.96}^{+1.06}$ \\
\hline 2MASXJ04234080+0408017 & $23.90_{-0.04}^{+0.04}$ & $1.769_{-0.092}^{+0.083}$ & $\left(1.65_{l}^{+7.43}\right) \times 10^{-2}$ & $\left(3.95_{-1.70}^{+1.87}\right) \times 10^{-1}$ & $1.11_{-0.38}^{+0.57}$ \\
\hline CGCG420-015 & $23.98_{-0.05}^{+0.04}$ & $1.885_{-0.058}^{+0.057}$ & $\left(4.92_{-0.66}^{+0.83}\right) \times 10^{-1}$ & $1.14_{-0.27}^{+0.37}$ & $\left(3.72_{l}^{+5.05}\right) \times 10^{-1}$ \\
\hline ESO 033-G002 & $22.26_{-0.03}^{+0.03}$ & $2.173_{-0.061}^{+0.067}$ & $\left(9.41_{-2.41}^{+2.39}\right) \times 10^{-2}$ & $2.34_{-0.51}^{+0.64}$ & $\left(4.77_{-3.19}^{+3.33}\right) \times 10^{-1}$ \\
\hline LEDA178130 & $23.05_{-0.02}^{+0.03}$ & $1.667_{-0.023}^{+0.047}$ & $\left(6.41_{-2.11}^{+2.07}\right) \times 10^{-2}$ & $\left(2.75_{l}^{+12.0}\right) \times 10^{-2}$ & $\left(7.41_{-2.07}^{+2.21}\right) \times 10^{-1}$ \\
\hline 2MASXJ05081967+1721483 & $22.21_{-0.04}^{+0.04}$ & $1.738_{-0.059}^{+0.062}$ & $\left(1.44_{-0.31}^{+0.31}\right) \times 10^{-1}$ & $\left(4.90_{-2.44}^{+3.00}\right) \times 10^{-1}$ & $1.19_{-0.89}^{+0.88}$ \\
\hline NGC2110 & $22.58_{-0.01}^{+0.01}$ & $1.640_{-0.008}^{+0.010}$ & $\left(3.26_{-0.59}^{+0.59}\right) \times 10^{-2}$ & $\left(1.73_{l}^{+2.79}\right) \times 10^{-2}$ & $\left(4.77_{-1.61}^{+1.61}\right) \times 10^{-1}$ \\
\hline ESO 005-G004 & $24.23_{-0.14}^{+0.30}$ & $1.8^{f}$ & $\left(1.67_{-0.41}^{+1.50}\right) \times 10^{0}$ & $1.88_{-1.32}^{+2.39}$ & $1.07_{-0.79}^{+1.19}$ \\
\hline ESO 121-IG028 & $23.36_{-0.04}^{+0.04}$ & $1.832_{-0.086}^{+0.084}$ & $\left(5.98_{-4.14}^{+4.12}\right) \times 10^{-2}$ & $\left(6.93_{-2.78}^{+3.37}\right) \times 10^{-1}$ & $<2.10 \times 10^{-1}$ \\
\hline $\mathrm{MCG}+06-16-028$ & $24.15_{-0.06}^{+0.08}$ & $1.792_{-0.104}^{+0.157}$ & $\left(4.02_{-1.43}^{+1.90}\right) \times 10^{-1}$ & $\left(3.62_{-1.78}^{+2.01}\right) \times 10^{-1}$ & $\left(9.44_{-4.81}^{+6.46}\right) \times 10^{-1}$ \\
\hline LEDA96373 & $24.10^{f}$ & $1.957_{-0.078}^{+0.070}$ & $\left(8.05_{-1.28}^{+4.35}\right) \times 10^{-1}$ & $2.11_{-0.93}^{+2.13}$ & $3.39_{-1.48}^{+2.45}$ \\
\hline UGC3995A & $23.59_{-0.05}^{+0.06}$ & $1.737_{-0.080}^{+0.075}$ & $\left(1.48_{-0.52}^{+0.54}\right) \times 10^{-1}$ & $2.01_{-0.54}^{+0.71}$ & $\left(6.68_{-4.02}^{+5.12}\right) \times 10^{-1}$ \\
\hline Mrk 1210 & $23.43_{-0.03}^{+0.02}$ & $1.876_{-0.052}^{+0.050}$ & $\left(1.13_{-0.30}^{+0.29}\right) \times 10^{-1}$ & $1.65_{-0.31}^{+0.34}$ & $1.71_{-0.35}^{+0.41}$ \\
\hline MCG-01-22-006 & $23.30_{-0.03}^{+0.02}$ & $1.560_{-0.061}^{+0.064}$ & $\left(6.29_{-2.84}^{+2.84}\right) \times 10^{-2}$ & $\left(4.44_{-1.73}^{+2.06}\right) \times 10^{-1}$ & $\left(5.85_{-2.97}^{+3.37}\right) \times 10^{-1}$ \\
\hline MCG+11-11-032 & $23.07_{-0.09}^{+0.09}$ & $1.866_{-0.160}^{+0.167}$ & $\left(3.90_{l}^{+9.00}\right) \times 10^{-2}$ & $1.40_{-0.77}^{+1.27}$ & $<1.25 \times 10^{0}$ \\
\hline Mrk 18 & $23.11_{-0.13}^{+0.10}$ & $1.627_{-0.114}^{+0.201}$ & $\left(1.76_{-1.10}^{+1.14}\right) \times 10^{-1}$ & $\left(1.03_{l}^{+5.61}\right) \times 10^{-1}$ & $2.32_{-1.30}^{+2.65}$ \\
\hline IC 2461 & $22.86_{-0.06}^{+0.06}$ & $1.802_{-0.093}^{+0.097}$ & $\left(1.18_{-0.39}^{+0.40}\right) \times 10^{-1}$ & $\left(6.95_{-3.38}^{+4.35}\right) \times 10^{-1}$ & $<3.50 \times 10^{-1}$ \\
\hline MCG-01-24-012 & $22.97_{-0.03}^{+0.02}$ & $2.074_{-0.060}^{+0.061}$ & $\left(4.03_{-2.15}^{+2.15}\right) \times 10^{-2}$ & $1.29_{-0.31}^{+0.38}$ & $\left(5.78_{l}^{+15.2}\right) \times 10^{-2}$ \\
\hline 2MASXJ09235371-3141305 & $23.89_{-0.09}^{+0.08}$ & $1.866_{-0.176}^{+0.163}$ & $\left(5.54_{l}^{+8.06}\right) \times 10^{-2}$ & $\left(8.12_{-2.61}^{+3.88}\right) \times 10^{-1}$ & $\left(2.03_{l}^{+5.45}\right) \times 10^{-1}$ \\
\hline NGC2992 & $22.04_{-0.01}^{+0.02}$ & $1.724_{-0.018}^{+0.018}$ & $\left(7.96_{-1.06}^{+1.07}\right) \times 10^{-2}$ & $\left(1.31_{-0.66}^{+0.69}\right) \times 10^{-1}$ & $\left(6.84_{-2.73}^{+2.73}\right) \times 10^{-1}$ \\
\hline NGC3079 & $24.52_{-0.04}^{+0.04}$ & $2.017_{-0.114}^{+0.115}$ & $\left(3.83_{-1.92}^{+2.82}\right) \times 10^{-1}$ & $\left(2.09_{-0.62}^{+0.90}\right) \times 10^{-2}$ & $\left(7.23_{-3.70}^{+6.47}\right) \times 10^{-2}$ \\
\hline ESO 263-G013 & $23.87_{-0.04}^{+0.03}$ & $1.732_{-0.085}^{+0.085}$ & $\left(6.24_{l}^{+6.46}\right) \times 10^{-2}$ & $\left(8.42_{l}^{+15.2}\right) \times 10^{-2}$ & $1.30_{-0.58}^{+0.67}$ \\
\hline NGC3281 & $24.08_{-0.10}^{+0.09}$ & $1.622_{-0.032}^{+0.033}$ & $\left(1.09_{-0.13}^{+0.79}\right) \times 10^{0}$ & $3.72_{-1.64}^{+4.38}$ & $<1.20 \times 10^{-1}$ \\
\hline MCG +12-10-067 & $23.24_{-0.07}^{+0.07}$ & $1.923_{-0.147}^{+0.155}$ & $\left(8.10_{-6.00}^{+6.00}\right) \times 10^{-2}$ & $1.37_{-0.65}^{+0.99}$ & $1.75_{-0.79}^{+1.09}$ \\
\hline MCG $+06-24-008$ & $22.60_{-0.10}^{+0.08}$ & $1.564_{-0.046}^{+0.047}$ & $\left(8.68_{-5.28}^{+5.32}\right) \times 10^{-2}$ & $<8.50 \times 10^{-2}$ & $1.09_{-0.94}^{+0.87}$ \\
\hline UGC5881 & $23.01_{-0.11}^{+0.10}$ & $1.628_{-0.149}^{+0.163}$ & $\left(1.37_{-0.80}^{+0.81}\right) \times 10^{-1}$ & $\left(7.08_{-5.04}^{+8.02}\right) \times 10^{-1}$ & $3.97_{-1.84}^{+2.78}$ \\
\hline NGC3393 & $24.38_{-0.05}^{+0.04}$ & $1.850_{-0.134}^{+0.140}$ & $\left(6.89_{-3.37}^{+4.01}\right) \times 10^{-1}$ & $\left(3.42_{-2.16}^{+2.41}\right) \times 10^{-2}$ & $\left(2.14_{-1.19}^{+1.94}\right) \times 10^{-1}$ \\
\hline Mrk 728 & $21.86_{l}^{+0.40}$ & $1.591_{-0.050}^{+0.055}$ & $\left(8.48_{-4.55}^{+4.52}\right) \times 10^{-2}$ & $<2.00 \times 10^{-1}$ & $\ldots$ \\
\hline 2MASXJ11364205-6003070 & $20.59_{-0.59}^{+0.48}$ & $1.996_{-0.074}^{+0.085}$ & $\left(1.13_{-0.42}^{+0.41}\right) \times 10^{-1}$ & $1.49_{-0.50}^{+0.67}$ & $\ldots$ \\
\hline NGC3786 & $22.52_{-0.42}^{+0.23}$ & $1.754_{-0.185}^{+0.203}$ & $\left(1.41_{-1.08}^{+1.10}\right) \times 10^{-1}$ & $\left(7.27_{-6.81}^{+11.5}\right) \times 10^{-1}$ & $6.94_{l}^{+7.26}$ \\
\hline NGC4388 & $23.67_{-0.02}^{+0.02}$ & $1.699_{-0.015}^{+0.016}$ & $\left(3.00_{-0.30}^{+0.30}\right) \times 10^{-1}$ & $\left(9.05_{-5.63}^{+6.15}\right) \times 10^{-2}$ & $6.82_{-0.55}^{+0.56}$ \\
\hline LEDA170194 & $22.75_{-0.07}^{+0.07}$ & $1.719_{-0.072}^{+0.090}$ & $\left(1.94_{-0.62}^{+0.62}\right) \times 10^{-1}$ & $\left(1.54_{l}^{+3.33}\right) \times 10^{-1}$ & $3.65_{-1.24}^{+1.55}$ \\
\hline NGC4941 & $24.14_{-0.07}^{+0.07}$ & $1.738_{-0.149}^{+0.157}$ & $\left(9.28_{-3.12}^{+5.52}\right) \times 10^{-1}$ & $\left(2.10_{-1.48}^{+1.87}\right) \times 10^{-1}$ & $\left(9.80_{-5.27}^{+8.40}\right) \times 10^{-1}$ \\
\hline NGC4992 & $23.63_{-0.03}^{+0.03}$ & $1.570_{-0.053}^{+0.052}$ & $\left(1.80_{-0.48}^{+0.48}\right) \times 10^{-1}$ & $\left(6.98_{-1.84}^{+2.20}\right) \times 10^{-1}$ & $<1.20 \times 10^{-1}$ \\
\hline Mrk 248 & $23.03_{-0.05}^{+0.04}$ & $1.992_{-0.103}^{+0.102}$ & $\left(6.90_{-3.86}^{+3.90}\right) \times 10^{-2}$ & $1.19_{-0.49}^{+0.61}$ & $\left(3.27_{-2.03}^{+2.35}\right) \times 10^{-1}$ \\
\hline ESO 509-IG066 & $22.89_{-0.07}^{+0.06}$ & $1.704_{-0.114}^{+0.118}$ & $\left(1.81_{-0.45}^{+0.46}\right) \times 10^{-1}$ & $\left(4.89_{-3.55}^{+4.81}\right) \times 10^{-1}$ & $1.34_{-0.67}^{+0.79}$ \\
\hline NGC5252 & $22.55_{-0.07}^{+0.06}$ & $1.662_{-0.023}^{+0.023}$ & $\left(8.17_{-2.51}^{+2.53}\right) \times 10^{-2}$ & $<2.50 \times 10^{-2}$ & $\ldots$ \\
\hline NGC5273 & $22.43_{-0.04}^{+0.03}$ & $1.797_{-0.046}^{+0.049}$ & $\left(1.10_{-0.21}^{+0.20}\right) \times 10^{-1}$ & $1.07_{-0.24}^{+0.29}$ & $3.62_{-0.54}^{+0.59}$ \\
\hline NGC5674 & $22.66_{-0.04}^{+0.05}$ & $1.871_{-0.078}^{+0.086}$ & $\left(1.48_{-0.35}^{+0.36}\right) \times 10^{-1}$ & $\left(4.61_{-2.87}^{+3.82}\right) \times 10^{-1}$ & $1.41_{-0.42}^{+0.47}$ \\
\hline NGC5728 & $24.14_{-0.02}^{+0.02}$ & $1.636_{-0.044}^{+0.045}$ & $\left(3.92_{-0.65}^{+0.69}\right) \times 10^{-1}$ & $\left(1.75_{-0.35}^{+0.39}\right) \times 10^{-1}$ & $\left(4.73_{l}^{+10.1}\right) \times 10^{-2}$ \\
\hline IC $4518 \mathrm{~A}$ & $23.23_{-0.05}^{+0.06}$ & $1.996_{-0.085}^{+0.091}$ & $<7.40 \times 10^{-2}$ & $2.90_{-0.79}^{+1.20}$ & $1.53_{-0.56}^{+0.59}$ \\
\hline 2MASXJ15064412+0351444 & $22.30_{-0.08}^{+0.09}$ & $1.689_{-0.057}^{+0.057}$ & $<1.10 \times 10^{-1}$ & $<3.00 \times 10^{-1}$ & $<2.00 \times 10^{0}$ \\
\hline NGC5899 & $22.98_{-0.04}^{+0.04}$ & $1.903_{-0.077}^{+0.080}$ & $\left(1.34_{-0.35}^{+0.36}\right) \times 10^{-1}$ & $1.13_{-0.35}^{+0.45}$ & $\left(2.53_{-2.33}^{+2.62}\right) \times 10^{-1}$ \\
\hline MCG+11-19-006 & $23.25_{-0.09}^{+0.08}$ & $1.576_{-0.146}^{+0.150}$ & $\left(4.41_{l}^{+8.99}\right) \times 10^{-2}$ & $\left(4.97_{-3.95}^{+5.83}\right) \times 10^{-1}$ & $1.29_{-0.75}^{+1.10}$ \\
\hline MCG-01-40-001 & $22.81_{-0.06}^{+0.05}$ & $1.790_{-0.085}^{+0.087}$ & $\left(1.99_{-0.44}^{+0.45}\right) \times 10^{-1}$ & $\left(9.13_{-3.64}^{+4.57}\right) \times 10^{-1}$ & $4.45_{-1.00}^{+1.23}$ \\
\hline NGC5995 & $22.09_{-0.03}^{+0.03}$ & $1.992_{-0.044}^{+0.047}$ & $\left(1.65_{-0.24}^{+0.23}\right) \times 10^{-1}$ & $1.16_{-0.26}^{+0.31}$ & $2.72_{-0.74}^{+0.76}$ \\
\hline MCG+14-08-004 & $23.14_{-0.07}^{+0.08}$ & $1.696_{-0.086}^{+0.132}$ & $\left(1.91_{-0.77}^{+0.77}\right) \times 10^{-1}$ & $\left(1.10_{l}^{+3.86}\right) \times 10^{-1}$ & $<6.90 \times 10^{-1}$ \\
\hline
\end{tabular}


Table 3

(Continued)

\begin{tabular}{|c|c|c|c|c|c|}
\hline \multirow[b]{2}{*}{ Name } & \multicolumn{5}{|c|}{ NuSTAR parameters } \\
\hline & $\begin{array}{r}\log \left(\mathrm{N}_{\mathrm{H}}\right) \\
\left(\mathrm{cm}^{-2}\right)\end{array}$ & Gamma & $\begin{array}{l}\mathrm{EW}(\mathrm{Fe} \mathrm{K} \alpha) \\
\quad(\mathrm{keV})\end{array}$ & $\begin{array}{l}\text { Reflection } \\
\text { Parameter }\end{array}$ & $\begin{array}{c}\text { Unabs. PL } \\
\text { Normalization }\end{array}$ \\
\hline NGC6240 & $24.10_{-0.02}^{+0.02}$ & $1.705_{-0.047}^{+0.047}$ & $\left(1.54_{-0.42}^{+0.42}\right) \times 10^{-1}$ & $\left(2.55_{-0.65}^{+0.70}\right) \times 10^{-1}$ & $<1.50 \times 10^{-1}$ \\
\hline NGC6300 & $23.23_{-0.02}^{+0.02}$ & $1.897_{-0.030}^{+0.029}$ & $\left(4.35_{-1.80}^{+1.81}\right) \times 10^{-2}$ & $1.47_{-0.17}^{+0.18}$ & $<4.50 \times 10^{-2}$ \\
\hline MCG $+07-37-031$ & $22.56_{-0.05}^{+0.06}$ & $1.681_{-0.069}^{+0.072}$ & $\left(7.93_{-3.71}^{+3.67}\right) \times 10^{-2}$ & $\left(3.54_{-2.33}^{+2.89}\right) \times 10^{-1}$ & $2.59_{-0.73}^{+0.82}$ \\
\hline IC 4709 & $23.42_{-0.04}^{+0.05}$ & $1.927_{-0.073}^{+0.071}$ & $\left(1.55_{-0.41}^{+0.41}\right) \times 10^{-1}$ & $2.06_{-0.49}^{+0.59}$ & $<4.30 \times 10^{-1}$ \\
\hline ESO 103-G035 & $23.33_{-0.01}^{+0.01}$ & $1.965_{-0.022}^{+0.021}$ & $\left(8.25_{-1.35}^{+1.37}\right) \times 10^{-2}$ & $1.19_{-0.11}^{+0.11}$ & $<1.10 \times 10^{-2}$ \\
\hline 2MASXJ20183871+4041003 & $23.14_{-0.04}^{+0.05}$ & $1.699_{-0.087}^{+0.087}$ & $\left(1.53_{-0.40}^{+0.41}\right) \times 10^{-1}$ & $\left(9.81_{-3.35}^{+4.19}\right) \times 10^{-1}$ & $<3.00 \times 10^{0}$ \\
\hline MCG+04-48-002 & $23.95_{-0.08}^{+0.08}$ & $1.764_{-0.156}^{+0.146}$ & $\left(3.41_{-1.27}^{+1.72}\right) \times 10^{-1}$ & $\left(5.50_{-2.80}^{+3.71}\right) \times 10^{-1}$ & $\left(4.13_{l}^{+10.8}\right) \times 10^{-1}$ \\
\hline IC 5063 & $23.42_{-0.03}^{+0.02}$ & $1.799_{-0.050}^{+0.050}$ & $\left(1.19_{-0.25}^{+0.26}\right) \times 10^{-1}$ & $\left(8.18_{-1.85}^{+2.02}\right) \times 10^{-1}$ & $\left(5.18_{-1.61}^{+1.81}\right) \times 10^{-1}$ \\
\hline MCG+06-49-019 & $<21.00$ & $1.7^{f}$ & $\left(5.02_{-1.71}^{+1.73}\right) \times 10^{-1}$ & $\left(3.31_{-3.18}^{+3.49}\right) \times 10^{-1}$ & $\ldots$ \\
\hline MCG $+01-57-016$ & $<20.10$ & $1.850_{-0.051}^{+0.052}$ & $\left(8.28_{-4.50}^{+4.52}\right) \times 10^{-2}$ & $1.26_{-0.36}^{+0.41}$ & $\ldots$ \\
\hline NGC7582 & $23.45_{-0.05}^{+0.04}$ & $1.764_{-0.038}^{+0.038}$ & $\left(2.48_{-0.41}^{+0.42}\right) \times 10^{-1}$ & $5.43_{-0.99}^{+1.29}$ & $1.77_{-0.87}^{+0.97}$ \\
\hline 2MASXJ23303771+7122464 & $22.86_{-0.21}^{+0.15}$ & $1.665_{-0.156}^{+0.194}$ & $\left(1.63_{l}^{+8.47}\right) \times 10^{-2}$ & $\left(2.81_{l}^{+7.11}\right) \times 10^{-1}$ & $2.51_{l}^{+3.78}$ \\
\hline PKS 2331-240 & $20.81_{-0.06}^{+0.06}$ & $1.811_{-0.019}^{+0.020}$ & $\left(9.81_{-2.96}^{+2.99}\right) \times 10^{-2}$ & $<1.30 \times 10^{-2}$ & $\ldots$ \\
\hline
\end{tabular}

Note. Parameters from the NUSTAR modeling of B18: column density (Column 2), intrinsic power-law slope (Column 3), equivalent width of the Fe Ka line (Column 4), the absolute value of the pexrav reflection parameter (Column 5), and the normalization of the unabsorbed, exponentially cutoff power law (Column 6). Uncertainties given as $l$ indicate that the lower limit of the uncertainty is poorly constrained, despite the fit returning a best value for the parameter. Further details of the modeling are given in Section 3.1 .

luminosity has a slope of $1.11 \pm 0.13$. The intrinsic X-ray luminosity relation that we find is also mostly consistent with the literature relations within our confidence interval even without the additional comparison uncertainty due to differences in fitting methodology. The scatter relative to the fits is about a factor of 2 larger in Figure 3(a) (for the correlation with intrinsic $L_{\mathrm{X}}$ ) than in Figure 3(b) (with reflected $L_{\mathrm{X}}$ ).

\subsubsection{Implications of the Luminosity Correlations}

These analyses support the idea that the reflected X-ray and IR emission are more strongly correlated than the intrinsic X-ray and IR emission. The correlation between the offsets from the Type 1 AGN IR-X-ray relations (e.g., Chen et al. 2017) and the reflection parameter (color-scale in Figures 3(a); see also Section 4.2.1) suggests either that obscuration is responsible or that the relation reflects a physical link due to the processes affecting both. However, we do not find a correlation between column density and the $10-50 \mathrm{keV}$ luminosity (Figure 7(a); $\rho=-0.024 \pm$ $0.124 ; \log (p)=-0.073_{-0.441}^{+0.073}$ ), the IR-to-X-ray (intrinsic) ratio (Figure $7(\mathrm{~b}) ; \rho=0.12 \pm 0.12 ; \log (p)=-0.48_{-0.66}^{+0.48}$ ), the IR-to-X-ray (reflected) ratio (Figure 7(c); $\rho=0.12 \pm 0.11 ; \log$ $(p)=-0.45_{-0.56}^{+0.45}$ ), or the reflection parameter (Figure $7(\mathrm{~d}) ; \rho=$ $\left.0.082 \pm 0.065 ; \log (p)=-0.30_{-0.53}^{+0.30}\right)$. Therefore, it is unlikely that the X-ray reflection and IR emission correlation is merely due to the optical depth of obscuring material.

This suggests that, on average, both the reflected X-ray emission and IR luminosity have been processed by the same, or at least a closely related structure, classically described as the "torus," although the luminosity relations do not specifically imply a particular geometry. Nuclear luminosity, composed of X-rays from the corona and the tightly related optical/UV emission from the accretion disk (e.g., Steffen et al. 2006; Lusso \& Risaliti 2017), will interact with this structure. Some of the X-ray emission will be reflected by gas, and a fraction of the total luminosity (dominated by the optical/UV from the disk) will be absorbed and reprocessed into thermal emission from the dust that we observe in the IR. As a result, the correlations we have found between the emission traced by the reflected X-rays and the accretion luminosity reprocessed into the IR may provide insights into the structure with which the nuclear emission is interacting, as we discuss further below.

Our analysis has one further implication that will require more detailed modeling to fully investigate. The pexrav model of reflection off of an infinite slab implicitly assumes interaction with Compton-thick gas. This assumption combined with a common structure resulting in both the IR reprocessing and the X-ray reflection has one of two possible implications. Either the IR is due to reprocessing by Compton-thick material or there should be similar relations between parameters expressing the interaction of the nuclear emission with the surrounding Compton-thick and Compton-thin gas components. Because Compton-thin $\left(\log \left(N_{\mathrm{H}}\left[\mathrm{cm}^{-2}\right]\right) \simeq 22-24\right)$ obscuration is typically optically thick to the UV emission, which is then reprocessed into IR emission (e.g., Fabian et al. 2008), the second possibility appears to be the more likely scenario. This scenario implies that tori models that include a two-phase medium containing denser, often Compton-thick, clumps dispersed within a more diffuse medium (e.g., Nenkova et al. 2008; Hönig \& Kishimoto 2010; Feltre et al. 2012; Stalevski et al. 2012; Siebenmorgen et al. 2015) should have similar, or at least correlated, covering fractions for the clumps and diffuse media.

\subsection{Modeling the Distribution of Covering Fractions}

Having found that reflected X-ray luminosity and IR luminosity may both be associated with the same obscuring structure, we investigate the relation between the reflection parameter and the ratio of the intrinsic $10-50 \mathrm{keV}$ luminosity to the IR luminosity and the links of this relation to covering fraction. Previous studies (e.g., Yaqoob \& Murphy 2011) found that the ratio of IR-to-X-ray luminosities was relatively insensitive to column density. We find a consistent lack of a 
Table 4

Luminosities

\begin{tabular}{|c|c|c|c|c|c|c|c|}
\hline \multirow[b]{2}{*}{ Name } & \multicolumn{6}{|c|}{ 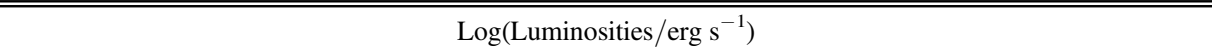 } & \multirow[b]{2}{*}{$\mathrm{f}_{\mathrm{AGN} I \mathrm{IR}}$} \\
\hline & $\begin{array}{l}2-10 \mathrm{keV} \\
\text { Observ. }\end{array}$ & $\begin{array}{l}10-50 \mathrm{keV} \\
\text { Observ. }\end{array}$ & $\begin{array}{l}2-10 \mathrm{keV} \\
\text { Intrinsic }\end{array}$ & $\begin{array}{l}10-50 \mathrm{keV} \\
\text { Intrinsic }\end{array}$ & AGN IR & Total IR & \\
\hline LEDA136991 & $39.78_{-0.03}^{+2.44}$ & $42.25_{-0.03}^{+0.03}$ & $41.85_{-0.30}^{+0.26}$ & $42.05_{-0.29}^{+0.24}$ & $43.06_{-0.11}^{+0.07}$ & $43.21_{-0.05}^{+0.04}$ & $0.70_{-0.11}^{+0.11}$ \\
\hline NGC262 & $41.77_{-0.01}^{+0.02}$ & $43.77_{-0.01}^{+0.02}$ & $43.50_{-0.02}^{+0.02}$ & $43.68_{-0.01}^{+0.01}$ & $43.82_{-0.07}^{+0.07}$ & $43.94_{-0.04}^{+0.04}$ & $0.76_{-0.10}^{+0.10}$ \\
\hline ESO 195-IG021 & $41.51_{-0.02}^{+0.01}$ & $43.82_{-0.01}^{+0.03}$ & $43.64_{-0.07}^{+0.07}$ & $43.73_{-0.05}^{+0.05}$ & $43.89_{-0.24}^{+0.18}$ & $44.38_{-0.02}^{+0.02}$ & $0.33_{-0.15}^{+0.14}$ \\
\hline IC 1663 & $39.89_{-0.01}^{+0.46}$ & $42.05_{--0.0}^{+0.36}$ & $41.79_{-0.18}^{+0.19}$ & $42.09_{-0.14}^{+0.08}$ & $43.61_{-0.06}^{+0.05}$ & $43.86_{-0.02}^{+0.02}$ & $0.57_{-0.10}^{+0.10}$ \\
\hline NGC513 & $40.78_{-0.02}^{+0.02}$ & $42.92_{-0.01}^{+0.03}$ & $42.53_{-0.11}^{+0.10}$ & $42.74_{-0.08}^{+0.06}$ & $<43.23$ & $44.23_{-0.02}^{+0.01}$ & $<0.10$ \\
\hline MCG-01-05-047 & $40.68_{-0.02}^{+0.02}$ & $42.96_{-0.02}^{+0.03}$ & $42.45_{-0.11}^{+0.11}$ & $42.59_{-0.09}^{+0.09}$ & $43.71_{-0.16}^{+0.13}$ & $44.24_{-0.02}^{+0.01}$ & $0.29_{-0.10}^{+0.10}$ \\
\hline NGC788 & $40.48_{-0.02}^{+0.01}$ & $42.95_{-0.02}^{+0.01}$ & $42.84_{-0.07}^{+0.06}$ & $43.00_{-0.06}^{+0.05}$ & $43.56_{-0.04}^{+0.04}$ & $43.62_{-0.03}^{+0.03}$ & $0.86_{-0.10}^{+0.10}$ \\
\hline NGC1052 & $40.36_{-0.01}^{+0.02}$ & $41.95_{-0.01}^{+0.02}$ & $41.53_{-0.03}^{+0.03}$ & $41.87_{-0.01}^{+0.01}$ & $42.71_{-0.04}^{+0.04}$ & $42.79_{-0.03}^{+0.03}$ & $0.83_{-0.10}^{+0.10}$ \\
\hline 2MFGC 2280 & $39.54_{-0.06}^{+0.11}$ & $42.56_{-0.02}^{+0.07}$ & $42.79_{-0.15}^{+0.16}$ & $43.10_{-0.11}^{+0.11}$ & $<43.20$ & $43.74_{-0.02}^{+0.02}$ & $<0.19$ \\
\hline NGC1365 & $40.56_{-0.01}^{+0.02}$ & $42.43_{-0.02}^{+0.01}$ & $41.77_{-0.04}^{+0.04}$ & $41.84_{-0.03}^{+0.03}$ & $<44.09$ & $44.60_{-0.02}^{+0.02}$ & $<0.26$ \\
\hline 2MASXJ04234080+0408017 & $40.81_{-0.01}^{+0.13}$ & $43.84_{-0.02}^{+0.02}$ & $43.83_{-0.09}^{+0.08}$ & $43.99_{-0.06}^{+0.06}$ & $44.30_{-0.13}^{+0.09}$ & $44.50_{-0.03}^{+0.04}$ & $0.64_{-0.11}^{+0.14}$ \\
\hline CGCG420-015 & $40.73_{-0.02}^{+0.02}$ & $43.53_{-0.01}^{+0.02}$ & $43.46_{-0.09}^{+0.09}$ & $43.54_{-0.08}^{+0.08}$ & $44.32_{-0.07}^{+0.06}$ & $44.42_{-0.05}^{+0.04}$ & $0.79_{-0.10}^{+0.10}$ \\
\hline ESO 033-G002 & $41.37_{-0.02}^{+0.01}$ & $43.15_{-0.02}^{+0.01}$ & $42.95_{-0.07}^{+0.07}$ & $42.83_{-0.05}^{+0.05}$ & $43.82_{-0.06}^{+0.06}$ & $43.93_{-0.03}^{+0.03}$ & $0.77_{-0.11}^{+0.10}$ \\
\hline LEDA178130 & $41.66_{-0.01}^{+0.03}$ & $44.02_{-0.01}^{+0.07}$ & $43.82_{-0.03}^{+0.03}$ & $44.05_{-0.03}^{+0.01}$ & $43.91_{-0.05}^{+0.06}$ & $43.97_{-0.04}^{+0.04}$ & $0.86_{-0.10}^{+0.10}$ \\
\hline 2MASXJ05081967+1721483 & $41.27_{-0.01}^{+0.01}$ & $43.16_{-0.02}^{+0.02}$ & $42.86_{-0.06}^{+0.06}$ & $43.05_{-0.04}^{+0.04}$ & $43.47_{-0.46}^{+0.23}$ & $44.08_{-0.03}^{+0.03}$ & $0.24_{-0.15}^{+0.22}$ \\
\hline NGC2110 & $42.24_{-0.01}^{+0.02}$ & $43.79_{-0.01}^{+0.01}$ & $43.60_{-0.02}^{+0.01}$ & $43.85_{-0.02}^{+0.01}$ & $43.12_{-0.21}^{+0.12}$ & $43.80_{-0.03}^{+0.02}$ & $0.20_{-0.10}^{+0.10}$ \\
\hline ESO 005-G004 & $39.51_{-0.07}^{+0.01}$ & $41.74_{-0.05}^{+0.02}$ & $41.41_{-0.44}^{+0.51}$ & $41.55_{-0.39}^{+0.47}$ & $<42.68$ & $43.68_{-0.01}^{+0.01}$ & $<0.10$ \\
\hline ESO 121-IG028 & $41.14_{-0.02}^{+0.01}$ & $43.72_{-0.01}^{+0.02}$ & $43.53_{-0.08}^{+0.08}$ & $43.65_{-0.05}^{+0.05}$ & $43.36_{-0.17}^{+0.17}$ & $43.63_{-0.07}^{+0.06}$ & $0.53_{-0.13}^{+0.11}$ \\
\hline $\mathrm{MCG}+06-16-028$ & $39.98_{-0.05}^{+0.07}$ & $42.66_{-0.03}^{+0.05}$ & $42.86_{-0.15}^{+0.16}$ & $43.01_{-0.13}^{+0.12}$ & $43.56_{-0.16}^{+0.10}$ & $44.09_{-0.03}^{+0.02}$ & $0.30_{-0.10}^{+0.10}$ \\
\hline LEDA96373 & $40.68_{-0.02}^{+0.02}$ & $43.38_{-0.02}^{+0.02}$ & $43.20_{-0.22}^{+0.18}$ & $43.23_{-0.21}^{+0.17}$ & $>44.57$ & $<44.61$ & $>0.90$ \\
\hline UGC3995A & $40.54_{-0.01}^{+0.02}$ & $42.99_{-0.02}^{+0.02}$ & $42.55_{-0.10}^{+0.09}$ & $42.74_{-0.08}^{+0.07}$ & $43.34_{-0.28}^{+0.24}$ & $43.85_{-0.05}^{+0.05}$ & $0.30_{-0.10}^{+0.10}$ \\
\hline Mrk 1210 & $41.04_{-0.02}^{+0.02}$ & $43.20_{-0.02}^{+0.02}$ & $42.90_{-0.05}^{+0.05}$ & $42.99_{-0.04}^{+0.04}$ & $44.08_{-0.09}^{+0.08}$ & $44.15_{-0.07}^{+0.06}$ & $0.84_{-0.10}^{+0.10}$ \\
\hline MCG-01-22-006 & $41.10_{-0.01}^{+0.01}$ & $43.49_{-0.01}^{+0.02}$ & $43.14_{-0.06}^{+0.06}$ & $43.45_{-0.04}^{+0.04}$ & $42.98_{-0.26}^{+0.21}$ & $43.87_{-0.02}^{+0.02}$ & $0.13_{-0.10}^{+0.10}$ \\
\hline $\mathrm{MCG}+11-11-032$ & $41.39_{-0.02}^{+0.04}$ & $43.83_{-0.02}^{+0.04}$ & $43.53_{-0.17}^{+0.15}$ & $43.63_{-0.13}^{+0.10}$ & $43.50_{-0.07}^{+0.07}$ & $43.78_{-0.03}^{+0.02}$ & $0.52_{-0.10}^{+0.10}$ \\
\hline Mrk 18 & $40.12_{-0.02}^{+0.06}$ & $42.02_{-0.01}^{+0.02}$ & $41.76_{-0.13}^{+0.15}$ & $42.02_{-0.10}^{+0.06}$ & $43.40_{-0.35}^{+0.24}$ & $43.68_{-0.03}^{+0.03}$ & $0.52_{-0.44}^{+0.29}$ \\
\hline IC 2461 & $40.62_{-0.02}^{+0.01}$ & $42.02_{-0.01}^{+0.03}$ & $42.23_{-0.09}^{+0.09}$ & $42.36_{-0.06}^{+0.06}$ & $42.42_{-0.21}^{+0.25}$ & $43.03_{-0.02}^{+0.01}$ & $0.25_{-0.15}^{+0.10}$ \\
\hline MCG-01-24-012 & $41.52_{-0.01}^{+0.02}$ & $43.51_{-0.01}^{+0.01}$ & $43.38_{-0.06}^{+0.06}$ & $43.33_{-0.04}^{+0.04}$ & $43.75_{-0.07}^{+0.07}$ & $43.93_{-0.04}^{+0.04}$ & $0.66_{-0.10}^{+0.10}$ \\
\hline 2MASXJ09235371-3141305 & $40.80_{-0.03}^{+0.03}$ & $43.77_{-0.03}^{+0.04}$ & $43.72_{-0.15}^{+0.14}$ & $43.82_{-0.09}^{+0.08}$ & $42.92_{-0.34}^{+0.20}$ & $43.53_{-0.03}^{+0.03}$ & $0.24_{-0.11}^{+0.17}$ \\
\hline NGC2992 & $41.67_{-0.01}^{+0.02}$ & $43.14_{-0.01}^{+0.01}$ & $42.87_{-0.02}^{+0.02}$ & $43.07_{-0.01}^{+0.02}$ & $<43.57$ & $43.91_{-0.02}^{+0.02}$ & $<0.40$ \\
\hline NGC3079 & $39.45_{-0.09}^{+0.02}$ & $41.72_{-0.03}^{+0.01}$ & $43.09_{-0.14}^{+0.16}$ & $43.08_{-0.12}^{+0.14}$ & $<43.55$ & $44.55_{-0.01}^{+0.01}$ & $<0.10$ \\
\hline ESO 263-G013 & $40.67_{-0.02}^{+0.08}$ & $43.52_{-0.02}^{+0.08}$ & $43.57_{-0.08}^{+0.07}$ & $43.76_{-0.06}^{+0.04}$ & $>43.82$ & $<44.00$ & $>0.80$ \\
\hline NGC3281 & $40.35_{-0.02}^{+0.01}$ & $42.86_{-0.02}^{+0.02}$ & $42.22_{-0.26}^{+0.22}$ & $42.49_{-0.26}^{+0.22}$ & $43.83_{-0.14}^{+0.11}$ & $44.26_{-0.04}^{+0.03}$ & $0.38_{-0.10}^{+0.10}$ \\
\hline MCG+12-10-067 & $40.77_{-0.02}^{+0.03}$ & $43.20_{-0.02}^{+0.03}$ & $42.95_{-0.15}^{+0.14}$ & $43.01_{-0.11}^{+0.09}$ & $44.02_{-0.11}^{+0.10}$ & $44.43_{-0.02}^{+0.02}$ & $0.39_{-0.10}^{+0.10}$ \\
\hline $\mathrm{MCG}+06-24-008$ & $40.83_{-0.01}^{+0.02}$ & $42.98_{-0.02}^{+0.01}$ & $42.67_{-0.04}^{+0.04}$ & $42.98_{-0.02}^{+0.02}$ & $<42.98$ & $43.98_{-0.02}^{+0.01}$ & $<0.10$ \\
\hline UGC5881 & $40.49_{-0.01}^{+0.03}$ & $42.72_{-0.01}^{+0.05}$ & $42.33_{-0.15}^{+0.15}$ & $42.59_{-0.11}^{+0.09}$ & $43.66_{-0.24}^{+0.18}$ & $44.13_{-0.02}^{+0.02}$ & $0.34_{-0.17}^{+0.17}$ \\
\hline NGC3393 & $39.85_{-0.08}^{+0.06}$ & $42.76_{-0.03}^{+0.04}$ & $43.50_{-0.13}^{+0.15}$ & $43.61_{-0.09}^{+0.11}$ & $43.64_{-0.07}^{+0.07}$ & $43.93_{-0.03}^{+0.03}$ & $0.51_{-0.10}^{+0.10}$ \\
\hline Mrk 728 & $41.16_{-0.02}^{+0.02}$ & $43.34_{-0.02}^{+0.02}$ & $43.04_{-0.04}^{+0.04}$ & $43.33_{-0.01}^{+0.02}$ & $>42.91$ & $<43.28$ & $>0.49$ \\
\hline 2MASXJ11364205-6003070 & $40.91_{-0.01}^{+0.01}$ & $42.60_{-0.02}^{+0.01}$ & $42.36_{-0.09}^{+0.08}$ & $42.36_{-0.07}^{+0.06}$ & $42.91_{-0.38}^{+0.24}$ & $43.63_{-0.03}^{+0.03}$ & $0.20_{-0.11}^{+0.14}$ \\
\hline NGC3786 & $40.09_{-0.02}^{+0.05}$ & $41.65_{-0.03}^{+0.07}$ & $41.49_{-0.18}^{+0.18}$ & $41.66_{-0.13}^{+0.11}$ & $<42.76$ & $43.51_{-0.03}^{+0.03}$ & $<0.16$ \\
\hline NGC4388 & $40.63_{-0.02}^{+0.01}$ & $42.82_{-0.01}^{+0.01}$ & $42.27_{-0.02}^{+0.02}$ & $42.48_{-0.02}^{+0.02}$ & $43.00_{-0.18}^{+0.16}$ & $43.58_{-0.03}^{+0.03}$ & $0.26_{-0.11}^{+0.10}$ \\
\hline LEDA170194 & $41.00_{-0.01}^{+0.03}$ & $43.26_{-0.02}^{+0.09}$ & $43.02_{-0.07}^{+0.07}$ & $43.22_{-0.05}^{+0.04}$ & $43.85_{-0.11}^{+0.09}$ & $44.16_{-0.03}^{+0.02}$ & $0.48_{-0.13}^{+0.10}$ \\
\hline NGC4941 & $39.40_{-0.03}^{+0.18}$ & $41.39_{-0.02}^{+0.13}$ & $41.73_{-0.16}^{+0.16}$ & $41.91_{-0.12}^{+0.11}$ & $42.36_{-0.05}^{+0.05}$ & $42.69_{-0.02}^{+0.02}$ & $0.47_{-0.10}^{+0.10}$ \\
\hline NGC4992 & $40.86_{-0.02}^{+0.01}$ & $43.55_{-0.01}^{+0.02}$ & $43.22_{-0.06}^{+0.05}$ & $43.52_{-0.05}^{+0.04}$ & $43.55_{-0.06}^{+0.07}$ & $43.77_{-0.03}^{+0.03}$ & $0.59_{-0.10}^{+0.10}$ \\
\hline Mrk 248 & $41.41_{-0.01}^{+0.02}$ & $43.74_{-0.01}^{+0.02}$ & $43.55_{-0.10}^{+0.10}$ & $43.56_{-0.07}^{+0.07}$ & $44.21_{-0.40}^{+0.14}$ & $44.59_{-0.04}^{+0.03}$ & $0.41_{-0.14}^{+0.29}$ \\
\hline ESO 509-IG066 & $41.43_{-0.01}^{+0.02}$ & $43.77_{-0.02}^{+0.04}$ & $43.47_{-0.11}^{+0.11}$ & $43.68_{-0.07}^{+0.07}$ & $43.97_{-0.19}^{+0.15}$ & $44.46_{-0.03}^{+0.04}$ & $0.34_{-0.11}^{+0.12}$ \\
\hline NGC5252 & $41.46_{-0.01}^{+0.02}$ & $43.49_{-0.02}^{+0.02}$ & $43.25_{-0.02}^{+0.03}$ & $43.49_{-0.01}^{+0.02}$ & $43.54_{-0.11}^{+0.07}$ & $43.80_{-0.03}^{+0.03}$ & $0.54_{-0.10}^{+0.11}$ \\
\hline NGC5273 & $40.82_{-0.02}^{+0.01}$ & $42.05_{-0.02}^{+0.02}$ & $41.74_{-0.04}^{+0.05}$ & $41.88_{-0.03}^{+0.03}$ & $41.48_{-0.27}^{+0.19}$ & $42.25_{-0.02}^{+0.02}$ & $0.15_{-0.10}^{+0.11}$ \\
\hline NGC5674 & $41.34_{-0.02}^{+0.01}$ & $43.35_{-0.01}^{+0.03}$ & $43.18_{-0.08}^{+0.08}$ & $43.27_{-0.06}^{+0.05}$ & $<43.38$ & $44.38_{-0.01}^{+0.01}$ & $<0.10$ \\
\hline NGC5728 & $40.10_{-0.01}^{+0.02}$ & $42.93_{-0.02}^{+0.01}$ & $42.84_{-0.04}^{+0.04}$ & $43.10_{-0.03}^{+0.03}$ & $42.97_{-0.37}^{+0.27}$ & $43.74_{-0.02}^{+0.02}$ & $0.18_{-0.14}^{+0.14}$ \\
\hline IC $4518 \mathrm{~A}$ & $40.87_{-0.01}^{+0.01}$ & $43.04_{-0.02}^{+0.02}$ & $42.68_{-0.11}^{+0.10}$ & $42.68_{-0.09}^{+0.08}$ & $44.03_{-0.30}^{+0.17}$ & $44.41_{-0.04}^{+0.03}$ & $0.41_{-0.23}^{+0.23}$ \\
\hline 2MASXJ15064412+0351444 & $40.83_{-0.02}^{+0.02}$ & $43.01_{-0.03}^{+0.03}$ & $42.80_{-0.05}^{+0.05}$ & $43.02_{-0.03}^{+0.03}$ & $<42.97$ & $43.26_{-0.03}^{+0.03}$ & $<0.42$ \\
\hline NGC5899 & $40.71_{-0.01}^{+0.02}$ & $42.41_{-0.02}^{+0.01}$ & $42.20_{-0.07}^{+0.08}$ & $42.27_{-0.05}^{+0.05}$ & $<43.46$ & $44.09_{-0.01}^{+0.02}$ & $<0.19$ \\
\hline MCG+11-19-006 & $40.81_{-0.01}^{+0.06}$ & $43.49_{-0.02}^{+0.07}$ & $43.13_{-0.14}^{+0.13}$ & $43.43_{-0.10}^{+0.08}$ & $43.84_{-0.13}^{+0.11}$ & $44.31_{-0.03}^{+0.03}$ & $0.35_{-0.10}^{+0.10}$ \\
\hline MCG-01-40-001 & $41.02_{-0.02}^{+0.01}$ & $43.14_{-0.01}^{+0.02}$ & $42.82_{-0.08}^{+0.08}$ & $42.97_{-0.06}^{+0.05}$ & $44.06_{-0.07}^{+0.05}$ & $44.31_{-0.02}^{+0.02}$ & $0.55_{-0.10}^{+0.10}$ \\
\hline NGC5995 & $41.60_{-0.01}^{+0.02}$ & $43.53_{-0.02}^{+0.01}$ & $43.32_{-0.04}^{+0.04}$ & $43.33_{-0.03}^{+0.03}$ & $44.29_{-0.13}^{+0.11}$ & $44.76_{-0.02}^{+0.02}$ & $0.34_{-0.10}^{+0.10}$ \\
\hline MCG+14-08-004 & $40.61_{-0.02}^{+0.06}$ & $42.83_{-0.02}^{+0.23}$ & $42.64_{-0.10}^{+0.10}$ & $42.85_{-0.08}^{+0.04}$ & $43.12_{-0.09}^{+0.08}$ & $43.28_{-0.05}^{+0.04}$ & $0.68_{-0.11}^{+0.10}$ \\
\hline
\end{tabular}


Table 4

(Continued)

\begin{tabular}{|c|c|c|c|c|c|c|c|}
\hline \multirow[b]{2}{*}{ Name } & \multicolumn{6}{|c|}{$\log \left(\right.$ Luminosities/erg s $\left.{ }^{-1}\right)$} & \multirow[b]{2}{*}{$\mathrm{f}_{\mathrm{AGN} \text { IR }}$} \\
\hline & $\begin{array}{l}2-10 \mathrm{keV} \\
\text { Observ. }\end{array}$ & $\begin{array}{l}10-50 \mathrm{keV} \\
\text { Observ. }\end{array}$ & $\begin{array}{l}2-10 \mathrm{keV} \\
\text { Intrinsic }\end{array}$ & $\begin{array}{l}10-50 \mathrm{keV} \\
\text { Intrinsic }\end{array}$ & AGN IR & Total IR & \\
\hline NGC6240 & $40.79_{-0.05}^{+0.04}$ & $43.69_{-0.02}^{+0.01}$ & $43.81_{-0.04}^{+0.05}$ & $44.02_{-0.02}^{+0.04}$ & $<45.21$ & $45.36_{-0.03}^{+0.02}$ & $<0.67$ \\
\hline NGC6300 & $40.79_{-0.01}^{+0.02}$ & $42.30_{-0.01}^{+0.02}$ & $41.99_{-0.03}^{+0.03}$ & $42.06_{-0.02}^{+0.02}$ & $43.09_{-0.15}^{+0.13}$ & $43.65_{-0.02}^{+0.02}$ & $0.27_{-0.10}^{+0.10}$ \\
\hline $\mathrm{MCG}+07-37-031$ & $41.65_{-0.02}^{+0.01}$ & $43.99_{-0.02}^{+0.03}$ & $43.69_{-0.07}^{+0.06}$ & $43.91_{-0.05}^{+0.04}$ & $43.78_{-0.28}^{+0.18}$ & $44.18_{-0.03}^{+0.03}$ & $0.39_{-0.20}^{+0.21}$ \\
\hline IC 4709 & $40.81_{-0.01}^{+0.02}$ & $43.08_{-0.01}^{+0.02}$ & $42.78_{-0.08}^{+0.08}$ & $42.83_{-0.06}^{+0.06}$ & $43.49_{-0.06}^{+0.06}$ & $43.71_{-0.03}^{+0.03}$ & $0.61_{-0.10}^{+0.10}$ \\
\hline ESO 103-G035 & $41.43_{-0.02}^{+0.01}$ & $43.46_{-0.02}^{+0.01}$ & $43.28_{-0.02}^{+0.02}$ & $43.31_{-0.01}^{+0.02}$ & $44.09_{-0.10}^{+0.08}$ & $44.18_{-0.07}^{+0.06}$ & $0.81_{-0.10}^{+0.10}$ \\
\hline 2MASXJ20183871+4041003 & $40.80_{-0.01}^{+0.02}$ & $42.94_{-0.01}^{+0.02}$ & $42.58_{-0.09}^{+0.08}$ & $42.79_{-0.06}^{+0.05}$ & $>43.12$ & $<43.37$ & $>0.75$ \\
\hline MCG $+04-48-002$ & $40.06_{-0.01}^{+0.10}$ & $42.60_{-0.01}^{+0.03}$ & $42.56_{-0.15}^{+0.14}$ & $42.73_{-0.11}^{+0.09}$ & $<43.42$ & $44.42_{-0.02}^{+0.02}$ & $<0.10$ \\
\hline IC 5063 & $41.02_{-0.02}^{+0.02}$ & $43.10_{-0.02}^{+0.02}$ & $42.87_{-0.05}^{+0.05}$ & $43.01_{-0.03}^{+0.03}$ & $44.26_{-0.05}^{+0.06}$ & $44.33_{-0.04}^{+0.05}$ & $0.84_{-0.10}^{+0.10}$ \\
\hline MCG+06-49-019 & $40.37_{-0.02}^{+0.03}$ & $42.26_{-0.03}^{+0.07}$ & $41.97_{-0.21}^{+0.21}$ & $42.18_{-0.03}^{+0.03}$ & $42.95_{-0.11}^{+0.11}$ & $43.40_{-0.02}^{+0.02}$ & $0.35_{-0.10}^{+0.10}$ \\
\hline $\mathrm{MCG}+01-57-016$ & $41.04_{-0.02}^{+4.41}$ & $43.06_{-0.01}^{+0.02}$ & $42.73_{-0.05}^{+0.05}$ & $42.84_{-0.04}^{+0.04}$ & $43.89_{-0.12}^{+0.11}$ & $44.14_{-0.03}^{+0.03}$ & $0.57_{-0.16}^{+0.12}$ \\
\hline NGC7582 & $40.41_{-0.02}^{+0.02}$ & $42.40_{-0.02}^{+0.02}$ & $41.61_{-0.07}^{+0.07}$ & $41.77_{-0.07}^{+0.07}$ & $<43.84$ & $44.29_{-0.02}^{+0.02}$ & $<0.29$ \\
\hline 2MASXJ23303771+7122464 & $40.83_{-0.01}^{+0.33}$ & $43.20_{-0.02}^{+0.13}$ & $42.90_{-0.15}^{+0.16}$ & $43.14_{-0.11}^{+0.08}$ & $<43.30$ & $44.04_{-0.02}^{+0.02}$ & $<0.16$ \\
\hline PKS 2331-240 & $41.80_{-0.02}^{+0.01}$ & $43.93_{-0.01}^{+0.01}$ & $43.79_{-0.02}^{+0.02}$ & $43.92_{-0.01}^{+0.01}$ & $43.98_{-0.03}^{+0.04}$ & $44.12_{-0.02}^{+0.03}$ & $0.71_{-0.10}^{+0.10}$ \\
\hline
\end{tabular}

Note. Observed and intrinsic X-ray luminosities in the $2-10 \mathrm{keV}$ and $10-50 \mathrm{keV}$ bands derived from the X-ray fitting (Columns 2-5). Intrinsic luminosities are corrected for the effects of reflection as well as absorption. Reflected luminosities are calculated by multiplying the reflection parameter by the intrinsic luminosity. The AGN component of the IR luminosity from the decomposition of the SED is given in Column 6. Its fraction relative to the total IR luminosity from the fits (Column 7) is given in Column 8.

correlation between the luminosity ratio and column density (Figure $7(\mathrm{~b}) ; \rho=0.12 \pm 0.12 ; \log (p)=-0.48_{-0.66}^{+0.48}$ ) in our purely phenomenological modeling. We therefore investigate the constraints that our modeling imposes on the covering fraction distribution based on the relation between IR-to-X-ray luminosity ratios and the reflection parameter.

\subsubsection{Determining the Comparison Parameters: Reflection and Infrared Excess}

In Figure 3(a), the points are color-coded according to the logarithm of the reflection parameter. We find a correlation between the intrinsic and reflected X-ray emission $(\rho=$ $0.55 \pm 0.09 ; \log (p)=-5.26 \pm 1.59$ in luminosities; $\rho=$ $0.40 \pm 0.11 ; \log (p)=-3.02 \pm 1.45$ in fluxes). This correlation, combined with the relation between reflected X-ray and IR emission, results in a tendency for AGNs in the lower right sector of Figure 3(a) to have higher reflection parameters. To examine the relationship between reflection and IR emission another way, we calculate the ratio of the observed IR emission compared to the expectation from the Chen et al. (2017) relation, shown in Figure 3(a) and derived for Type 1 AGNs, to calculate the expected IR emission from the intrinsic hard $\mathrm{X}$-ray luminosity. We refer to this ratio as the IR excess:

$$
\begin{aligned}
& \log (\text { IR Excess })=\log \left(\frac{\text { Observed AGN IR }}{\text { Expected AGN IR }}\right), \text { where } \\
& \log (\text { Expected AGN IR })=\log \left(\mathrm{IR}_{\text {corr }}\right) \\
& +\left[\log \left(L_{10-50 \mathrm{keV}}\right)-C_{1}-\log \left(X_{\text {corr }}\right)\right] / \mathrm{C}_{2}+45 .,
\end{aligned}
$$

in which $\mathrm{IR}_{\text {corr. }}$ is the Mullaney et al. (2011) ratio between $6 \mu \mathrm{m}$ and total IR emission, and $X_{\text {corr. }}$ is the ratio between $2-10 \mathrm{keV}$ and $10-50 \mathrm{keV}$ luminosity, assuming a $\Gamma=1.8$ power law. Depending on whether the $\log \left(L_{10-50 \mathrm{keV}} / \mathrm{erg} \mathrm{s}^{-1}\right)$ is above or below 44.56 (corresponding to a $\log \left(L_{6 \mu \mathrm{m}}\right)=44.79$ ), $\left[C_{1}, C_{2}\right]$ is [44.51, 0.40] or [44.60, 0.84], respectively (Chen et al. 2017).
We plot IR excess against reflection parameter in Figure 4 $(\rho=0.51 \pm 0.11 ; \log (p)=-4.51 \pm 1.62){ }^{32}$

We fit this parameter pairing using our orthogonal regression methodology, thereby using the limits and uncertainties on both IR excess and reflection parameter simultaneously. The best-fit line is given by

$$
\begin{aligned}
\log \left(R_{\text {pex }}\right)= & -(0.17 \pm 0.21) \\
& +(1.12 \pm 0.37) \times \log (\text { IR Excess })
\end{aligned}
$$

\subsubsection{Modeling Observables from Covering Fractions}

We developed a simple model to explore the physical origin of this relation, in particular whether we can parameterize it solely on the basis of the covering fraction. With this model, we are implicitly assuming an axisymmetric geometry with relatively constant distribution of the obscuring matter, seen along random lines of sight. We generate a range of covering fraction distributions of all AGNs, including both wide and narrow Gaussian distributions and a uniform distribution (histograms of Figure 5) to cover the full range of possible scenarios. We step through central values of the Gaussian from 0 to 1 in steps of 0.05 and through values of FWHM from 0.1 to 2 in steps of 0.1 , for a total of 420 models.

For each distribution, we draw 10,000 simulated AGNs for which we set the probability of being classified as a Type 2 equal to the covering fraction (e.g., Elitzur 2012; Netzer 2015). We then separate the sample into Type 1 and Type 2 subsamples. For each of the simulated Type 2 AGNs, we calculate a model reflection parameter using:

$$
\log \left(R_{\text {pex }}\right)=1.7 \times f_{\text {cov }}-1.4
$$

where $f_{\text {cov }}$ is the covering fraction (i.e., the fraction of the sky obscured by gas and dust). This empirical relation is based on the determination of both reflection parameter and covering

\footnotetext{
32 The strength of this correlation is at a level similar to that between fluxes because the ratio divides out the luminosity distance.
} 


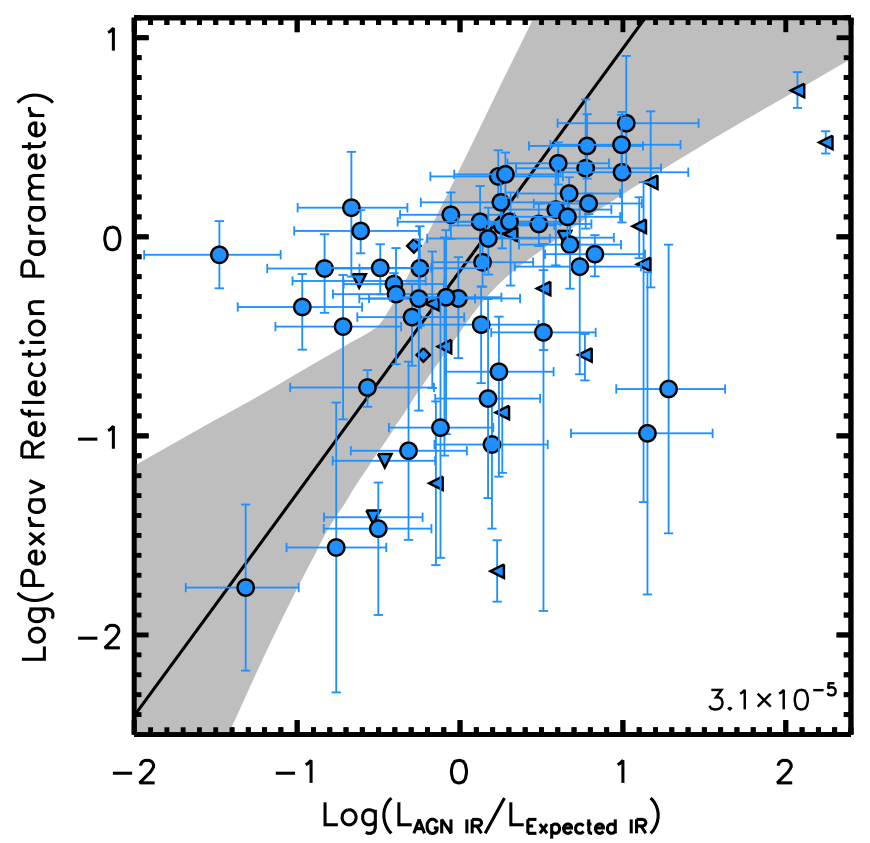

Figure 4. Excess IR luminosity (compared to the expectation from the intrinsic $10-50 \mathrm{keV}$ luminosity and the Chen et al. (2017) relation) vs. pexrav reflection. Limits $(3 \sigma)$ in either the IR luminosity (and therefore the IR excess) or the reflection parameter are shown as triangles, unless both are limits, in which case diamonds without error bars are used. The solid line shows the best fit including the censored data, with the $3 \sigma$ region of confidence for the fit derived from the bootstrapping analysis delineated by the gray-shaded region. There is a correlation between these parameters, whose Spearman rank correlation probability of the absence of a correlation is given in the lower right, which we used to probe the covering fraction distribution.

fraction for a larger sample of NUSTAR observed AGNs using more complicated models compared to fits using the phenomenological modeling used in this article (Baloković et al. 2018, in preparation). ${ }^{33}$ Baloković et al. (2018) showed the results of this modeling for four galaxies (their Figure 8), which support the linear scaling between $f_{\text {cov }}$ and $\log \left(R_{\text {pex }}\right)$. We also investigate the effect on our conclusions due to the variations on this relation (discussed in Section 4.2.5 and Appendix B).

Given the correlation between reflected and IR emission, we assume the degree of reflection (and absorption) depends on the covering fraction (e.g., Maiolino et al. 2007; Treister et al. 2008; Elitzur 2012). Therefore, we parameterize $L_{\mathrm{IR}}=\eta \times$ $f_{\text {cov }} \times L_{\mathrm{bol}} . \eta$ encompasses all other constants of proportionality, including an assumed constant ratio between the optical/ UV disk emission and the reprocessed IR emission that is the same for Type 1 and Type 2 AGNs. We use this to determine the relation between the IR excess and the covering fraction. Because our observed IR excess is determined relative to a relation derived for Type 1 AGNs, the intrinsic IR excess is given by the equation:

$$
\log (\text { IR Excess })=\log \left(f_{\text {cov }} /\left\langle f_{\text {cov; Type } 1}\right\rangle\right) .
$$

The average covering fraction for Type 1 AGNs is calculated from the Type 1 subsample of simulated AGNs. We investigate the robustness of this parametrization in two ways. First, we relax the assumption of a linear scaling between IR luminosity

\footnotetext{
33 As discussed in footnote 25 , there exists a degeneracy between assumed inclination and $R_{\text {pex }}$ in the pexrav model. This empirical relation was derived for values of $R_{\text {pex }}$ determined with a fixed inclination of $60^{\circ}$.
}

and covering fraction, using the IR-to-bolometric luminosity dependence on covering fraction of Stalevski et al. (2016). Second, we explore the effect of changing the dependence of the expected IR emission (and therefore the IR excess) on the observed intrinsic X-ray emission. These variations and their effect on our conclusions are discussed in Section 4.2.5 and Appendix B.

We add scatter to create mock observables for these model values. For the reflection parameter, the magnitude of the scatter is set by the average observed uncertainty of $\sim 0.3$ dex. For the IR excess, we find a scatter in IR-to-X-ray luminosity ratios relative to the literature relations (Figure 3(a)) of $\sim 0.4$ dex, similar if a bit larger than that found by Gandhi et al. (2009) in the MIR to X-ray luminosity ratio, as might be expected given the use of SED decomposition in S17 compared to the nuclear MIR fluxes used by Gandhi et al. (2009).

\subsubsection{Comparison of Models and Observations}

Figure 5 compares the results of a subset of our models to our observations. The blue points are the same as those in Figure 4, whereas the red contours show the distributions of the mock observables calculated using Equations (3) and (4) from the covering fraction of each simulated Type 2 AGNs. We quantify the likelihood that the observations are consistent with each set of mock observables using a two-dimensional KS (2D-KS) test (Peacock 1983; Goulding et al. 2014). We follow the methodology of Goulding et al. (2014) and run 10,000 bootstrap samples of the observations and mock observables for which we calculate the 2D-KS statistic and associated probability of the null hypothesis that both samples are consistent with having the same parent population.

Figure 6 shows the medians of the probability distribution resulting from each set of bootstrap runs. The three colored blocks at the upper right show the results for the uniform distribution. The larger block in the middle shows that this distribution has a 2D-KS median probability indicative that the null hypothesis cannot be rejected. We find that the probability distributions generated by the bootstrap methodology have a typical breadth of about $1 \mathrm{dex}$, as illustrated by the two color blocks to the left and right of the block corresponding to the uniform distribution's median probability. As a result, we use three color scales to indicate the likelihood of the null hypothesis. Models whose median 2D-KS probability is at least $10^{-3}$ are shown with blue colors. For these models, we cannot reject the null hypothesis that the data and mock observables are consistent. Models that significantly reject the null hypothesis by having at least $84 \%$ of their probability distribution (corresponding to all probabilities less than the median $+1 \sigma$ probability) less than $10^{-3}$ are shown with red colors. The intermediate set of models, shown in purple, have a median probability less than $10^{-3}$, but the standard deviation of its probability distribution extends above $10^{-3}$. For this set of models, it is possible that the null hypothesis is not rejected, because models shown in very light blue or dark purple have a very similar probability.

\subsubsection{Implications for Covering Fraction Distributions}

Taken together, Figures 5 and 6 provide some insight into the underlying distribution of covering fractions for all AGNs. Narrow Gaussian models for distributions of covering fractions (e.g., Figures 5(b), (c), or (f)) tend to poorly match the 

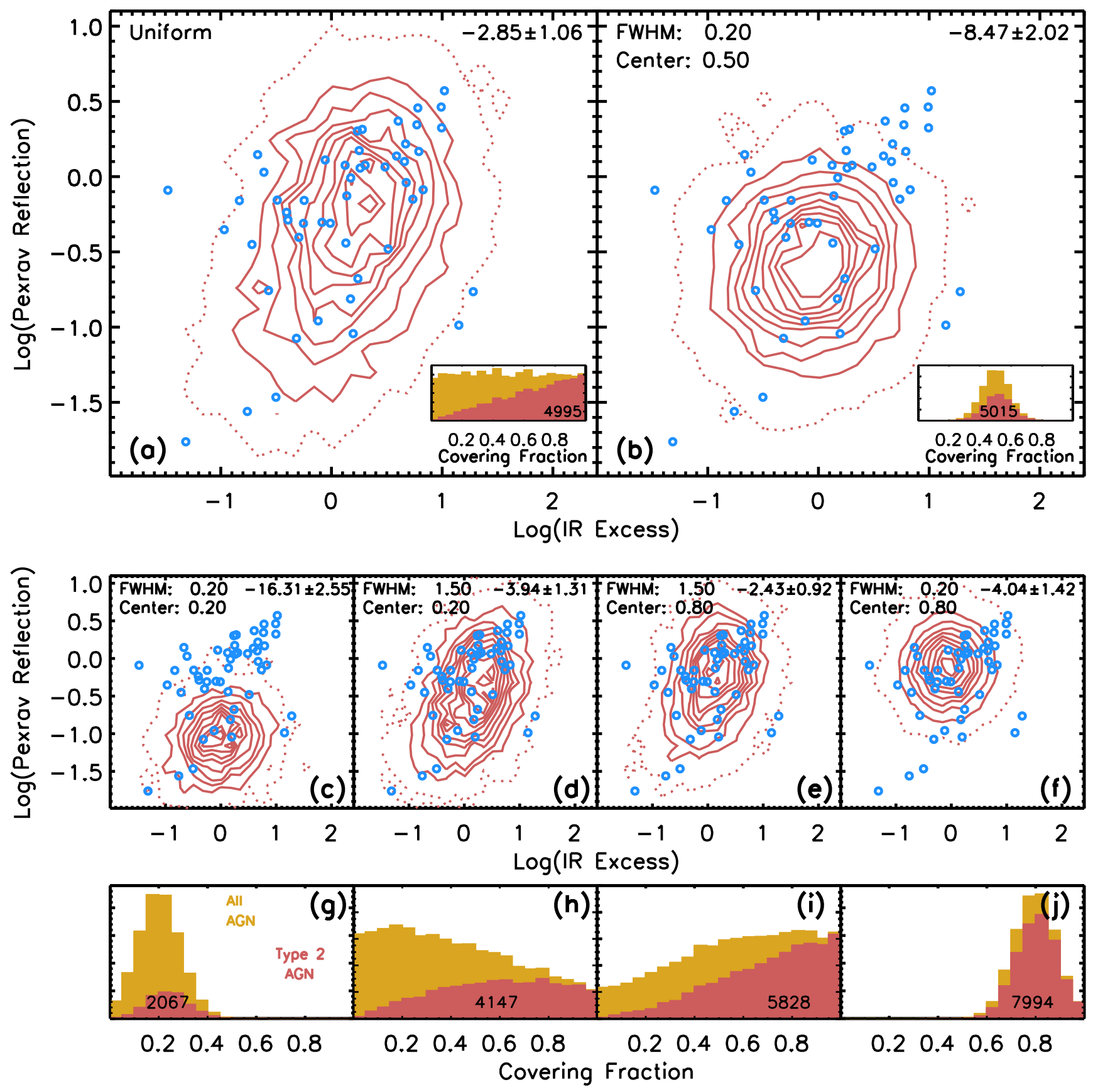

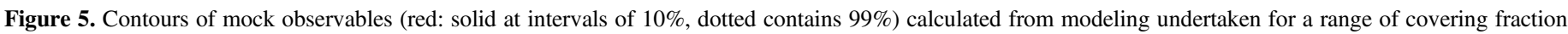

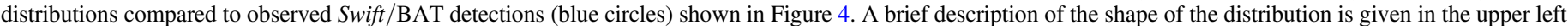

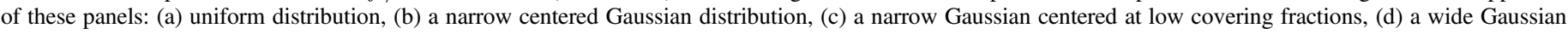

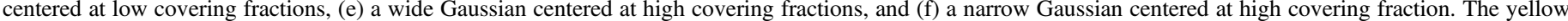

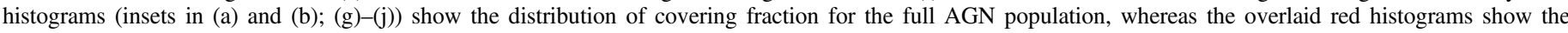

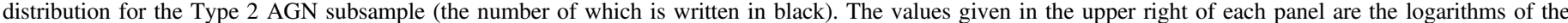

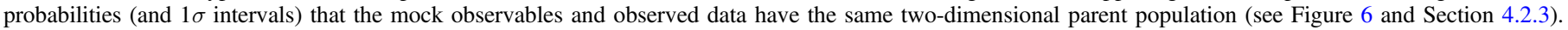

observations. This is particularly acute for narrow distributions skewed to peak at low covering fractions (e.g., Figure 5(c)). Even very wide distributions skewed to peak at low covering fractions (e.g., Figure 5(d)) at best have only marginal or suggestive indications of agreement with the observations. Broad, centered (e.g., Figure 5(a)) or peaking at high covering fraction (e.g., Figure 5(e)) result in observables that match the data best. We also find that once the distribution has a FWHM of 1.0, a further increase in breadth does not tend to change the degree of agreement. The narrowest distributions whose observables are consistent with the data are centered at covering fractions of $\sim 0.70-0.80$.

Broad distributions of covering fraction, combined with the assumption that the likelihood of a Type 2 designation increases with covering fraction (e.g., Elitzur 2012), also have the benefit of yielding distributions of the Type 2 covering fraction similar to what has been observed with more complex modeling. Highspatial-resolution IR studies of small samples of local quasars have found that, although the distributions of covering fractions for Type 1 and 2 AGNs are different, they also overlap 


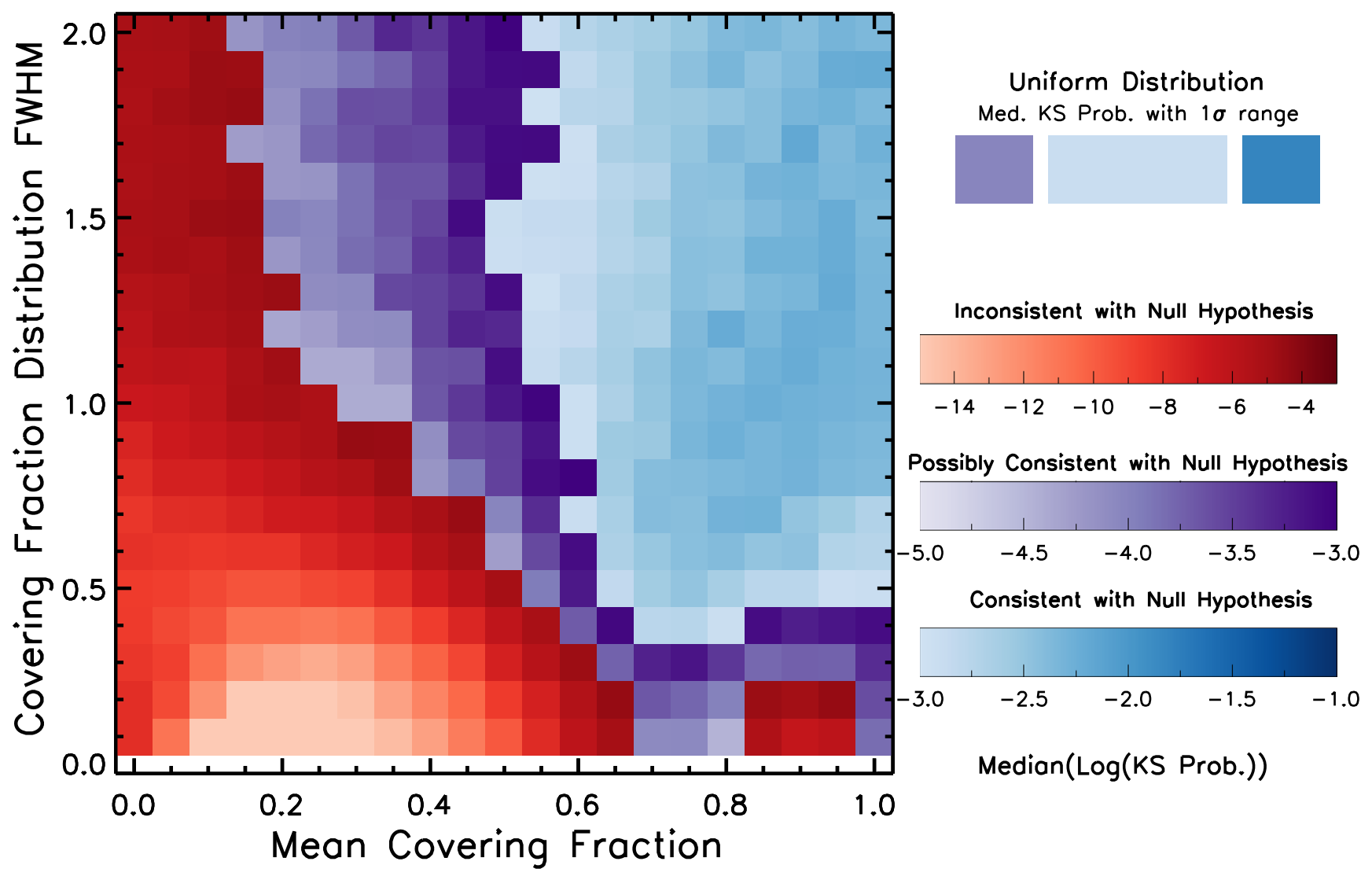

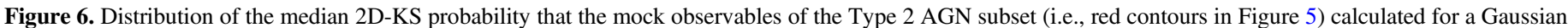

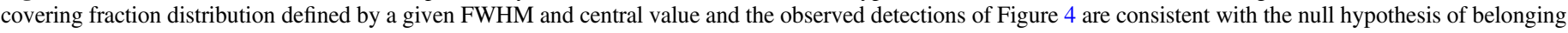

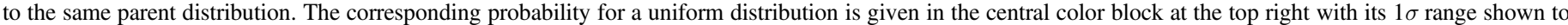

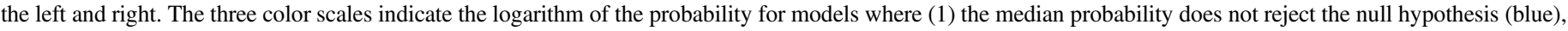

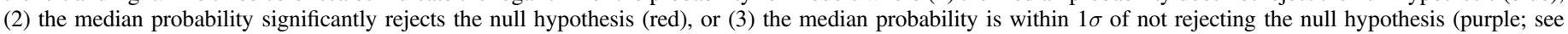
Section 4.2.3).

significantly (e.g., Mor et al. 2009; Ramos Almeida et al. 2011; Alonso-Herrero et al. 2011; Ichikawa et al. 2015). Mateos et al. (2016) recently undertook clumpy torus modeling of the NIR to MIR SEDs of 227 X-ray selected AGNs and likewise found broad, overlapping distributions for the covering fractions of both obscured and unobscured AGNs. Their distributions are different from Gaussians or a uniform distribution due to the presence of additional low covering fraction sources. However, our Type 2 subsets for broad distributions (e.g., Figure 5(a) inset or Figure 5(i)) show similar peaks at high covering fractions and decline with decreasing covering fraction as their Type 2 distribution (red line in their Figure 3). We used their distribution for all AGNs (black line in their Figure 3) to generate another set of mock observables. We find that the mock observables from this underlying distribution agree similarly well with our data to some of our very broad models (e.g., Figures 5(a), (d)-(e); log (probabilities) $=-3.53 \pm 1.24$ ), indicating that for broad distribution, this analysis is not sensitive to the details of their shape.

Figure 6 also shows that Gaussian distributions centered at covering fractions of $\sim 0.70-0.80$ can have a broad range of FWHM capable of reproducing the observations, down to relatively narrow widths. Interestingly, Ricci et al. (2015) recently showed that, on the basis of the obscured fractions in the BAT AGN Spectroscopic Survey, the typical covering fraction of AGNs should be approximately $70 \%$. Our analysis is consistent with these results, finding that even relatively narrow distributions centered at $70 \%$ yield observables consistent with our data.

Despite this consistency with Ricci et al. (2017a) regarding typical covering fractions, our model predicts a different relationship between the strength of the reflection component and $N_{\mathrm{H}}$. Specifically, we expect Type 2 AGNs to have stronger reflection, because our modeling tends to give them higher covering fractions. Although we do not find a significant correlation between $R_{\text {pex }}$ and $N_{\mathrm{H}}$, the median and average $R_{\text {pex }}$ of our AGNs with $N_{\mathrm{H}} \geqslant 10^{23} \mathrm{~cm}^{-2}$ are larger than those for our AGNs with $N_{\mathrm{H}}<10^{23} \mathrm{~cm}^{-2}$. This is more consistent with the results of Ricci et al. (2011; see also Vasudevan et al. 2013; Esposito \& Walter 2016). One possible explanation for the closer similarity to the Ricci et al. (2011) results compared to the Ricci et al. (2017a) results may lie in a modeling degeneracy. In both our modeling and the Ricci et al. (2011) modeling, the typical $\Gamma$ is consistent across different bins of $N_{\mathrm{H}}$. However, in Ricci et al. (2017a), there is a significant difference in the distribution of the photon indices of the most obscured AGNs compared to the distribution for their less obscured AGNs. Because there exists a degeneracy between $\Gamma$ and the reflection parameter (e.g., Del Moro et al. 2017; see also Appendix A), some of the effect seen in Ricci et al. (2017a) may therefore be induced by the difference in $\Gamma$. 


\subsubsection{Robustness of the Modeling Results}

Given the phenomenological nature of the spectral modeling used in our analysis, we chose to use a simple model for our mock observables to limit the number of free parameters. As a result, there are multiple additional considerations that could be taken into account to further constrain the nature of the covering fraction distribution. For example, our model does not include obscuration or reflection due to dust in the polar regions (e.g., Hönig et al. 2012; López-Gonzaga et al. 2016). Although our model does not assume a geometry that precludes its presence, it may have a different heating mechanism that would not be captured in our model. Additionally, given the relatively small dynamic range of our luminosities, we also do not include a dependence of the covering fraction on AGN luminosity, the so-called receding torus models (e.g., Lawrence 1991; Simpson 2005), although recent results suggest that covering fraction may not vary significantly with luminosity (Mateos et al. 2017; Ichikawa et al. 2018).

As was mentioned in 4.2.2, the underlying uncertainty in determining covering fraction, including its dependence on other AGN properties, manifests in uncertainty in the empirical relations we use to calculate the mock observables of reflection parameter and IR excess. We explored two variations in each parameter to explore the robustness of our conclusions. Appendix B contains a detailed discussion of these alternative empirical relations for the reflection parameter as well as IR excess. The results of these tests are all consistent with our conclusions; specifically, we still find that broad distributions of covering fractions result in mock observables with the best agreement with our data and that the narrowest models yielding observables in agreement with our data tend to be centered around $\sim 70 \%$. The range of distributions yielding observables consistent with our observations show greater sensitivity to the relation between covering fraction and reflection parameter than to the relation between covering fraction and IR excess.

In determining the reflection parameter for our observations, the inclination of the pexrav was fixed to $60^{\circ}$ due to the degerenacy between the normalization of the reflection component and the inclination parameter (e.g., Dauser et al. 2016). Inclination does not affect the total IR emission of the torus (e.g., Stalevski et al. 2016), but it can affect the degree to which X-rays are reflected into the observed line of sight. As a result, inclination effects could be responsible for at least some of the scatter in Figure 4. However, disentangling this effect will require more complex modeling than that used in this analysis.

\section{Summary}

We performed joint IR and X-ray phenomenological modeling of a large sample of obscured AGNs. We found a significant correlation between the reflected X-ray and IR emission, with multiple suggestive indications that this correlation is stronger than that between intrinsic X-ray and IR emission. This relation suggests that both the X-ray reflection and the UV emission reprocessed into IR have been processed by the same structure.

We parametrized this effect as a covering fraction, encompassing both geometrical factors and the impact of clumpiness, and investigated which distributions of covering fractions can reproduce the observed distributions of IR excess and reflection parameters. A range of broad covering fraction distributions of the underlying total AGN population (e.g., Figures 5(a), (e)) results in mock observables, determined from simple empirical relations, consistent with our observations. We also find that the narrowest distributions resulting in observables in agreement with our data are centered around covering fractions of $70 \%-80 \%$. These results are consistent with both other methodologies for estimating covering fraction: the set of independent estimates of the covering fraction of individual objects suggests a broad distribution of covering fraction (e.g., Mateos et al. 2016), and statistical estimates of the typical covering fraction from sample properties (e.g., Ricci et al. 2015 ) find an expected covering fraction of $\sim 70 \%$.

Although our modeling was purposely kept simple to investigate how much can be gleaned without the use of complex assumptions, their implications regarding covering fraction distribution are not in agreement with the classical unification model (e.g., Antonucci 1993). In the simplest classical picture, all AGNs have the same covering fraction and opening angle, and it is only orientation that governs whether an AGN is identified as obscured. In contrast, in clumpy torus models (e.g., Nenkova et al. 2008; Stalevski et al. 2012), the covering fraction depends on the number and distributions of obscuring clouds, possibly embedded in a more diffuse medium. Our modeling suggests that the clumps and the more diffuse media should have at least correlated covering fraction, but more detailed modeling will be necessary to fully investigate this question.

We thank the referee for useful comments, which particularly strengthened the statistical analysis. L.L. and R.C.H. acknowledge support from NASA through grant No. NNX15AP24G. L.L. acknowledges support from NASA through grant No. NNX17AB58G. R.C.H acknowledges support from the National Science Foundation through CAREER grant No. 1554584. M.B. acknowledges support from NASA Headquarters under the NASA Earth and Space Science Fellowship Program, grant No. NNX14AQ07H, and support from the black hole Initiative, which is funded by a grant from the John Templeton Foundation. C.R. acknowledges the CONICYT+PAI (Convocatoria Nacional subvencion a instalacion en la academia convocatoria año 2017 PAI77170080) and financial support from FONDECYT 1141218, Basal-CATA PFB-06/2007, and the China-CONICYT fund. F.E.B. acknowledges support from CONICYT-Chile (BasalCATA PFB-06/2007, FONDECYT Regular 1141218), the Ministry of Economy, Development, and Tourism's Millennium Science Initiative through grant No. IC120009, awarded to The Millennium Institute of Astrophysics, MAS. M.K. acknowledges support from NASA through ADAP award No. NNH16CT03C. A.M. acknowledges support from the ASI/INAF through grant No. I/037/12/0-011/13. L.Z. acknowledges financial support under ASI/INAF contract No. I/037/12/0. This work made use of ASURV Rev. 1.2 (Lavalley et al. 1992), which implements the methods presented in Isobe et al. (1986).

Facilities: Herschel, NuSTAR, Swift, WISE.

\section{Appendix A \\ Relationships Between Other X-Ray and Infrared Properties}

Most pairings of IR and X-ray properties, beyond those discussed in Section 4 do not yield significant correlations. We show a subset that may be of interest in Figure 7. Of those with significant correlations, several are due to definitions of model parameters or model degeneracies. Over the luminosity range of our sample, the anticorrelation of the equivalent width of the Fe $\mathrm{K} \alpha$ line to the observed X-ray luminosities $(\rho=-0.50 \pm$ $0.10 ; \log (p)=-4.42 \pm 1.76)$ is primarily due to the reduction 

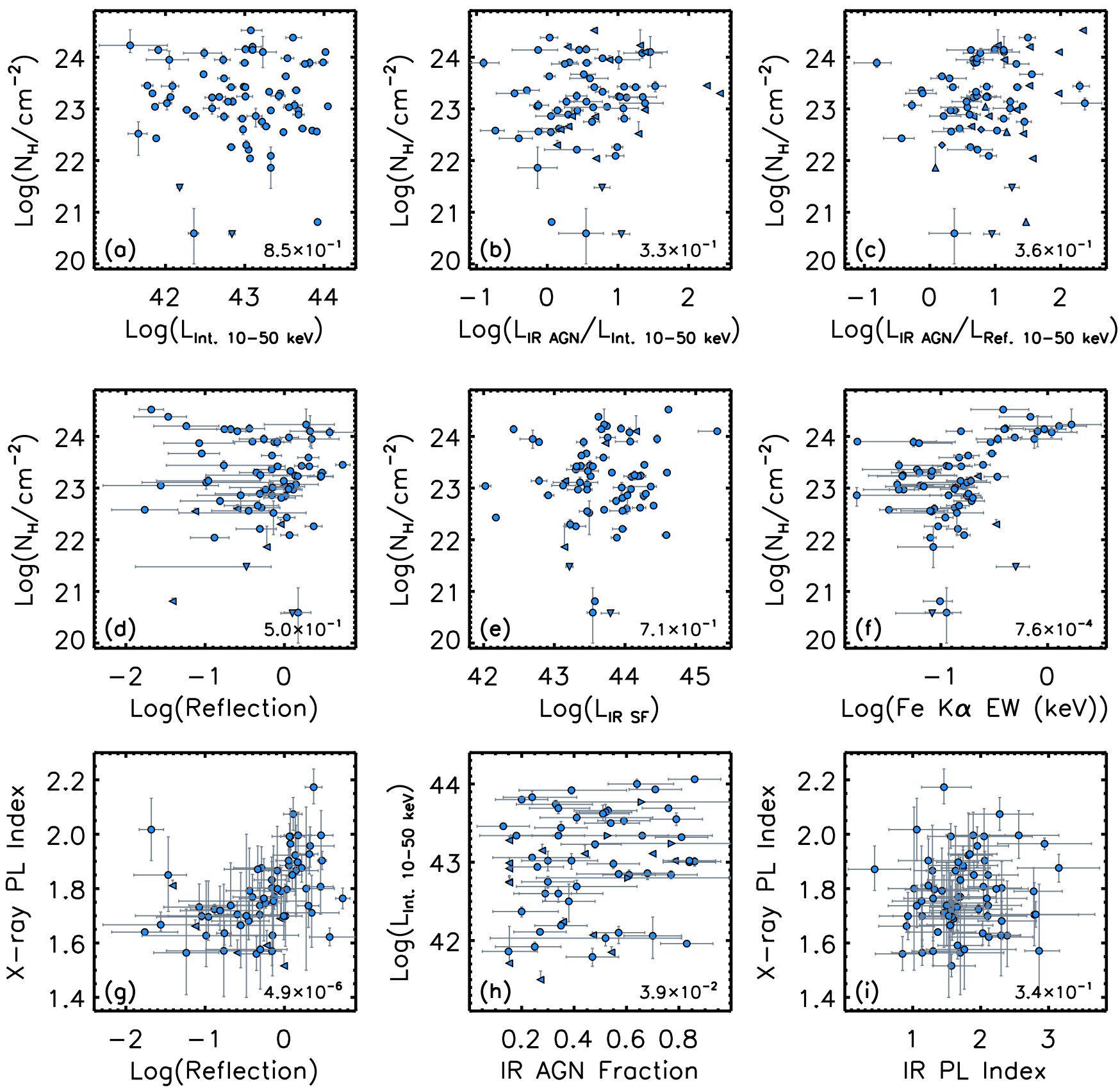

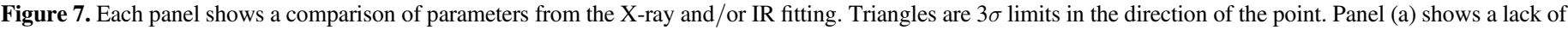

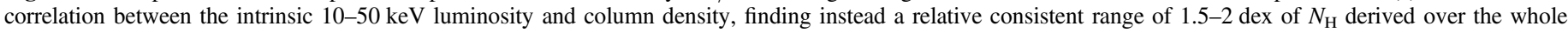

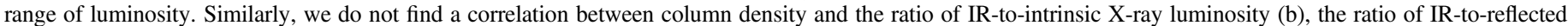

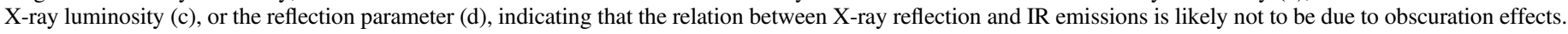

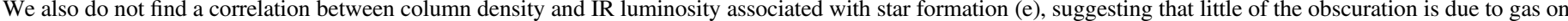

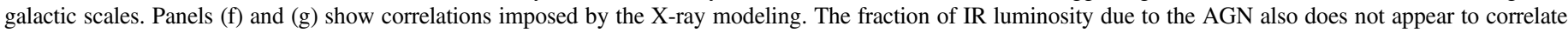

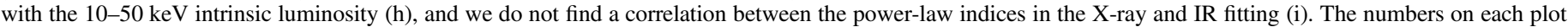
are the Spearman rank correlation probability of the absence of a correlation.

of the continuum level resulting in an increase in equivalent width even at constant line flux. This effect is also seen in the correlation between column density and $\mathrm{Fe} \mathrm{K} \alpha$ equivalent width (Figure 7(f); $\rho=0.41 \pm 0.12 ; \log (p)=-3.12 \pm 1.50)$, which is due to modeling methodology. As absorption increases, the continuum is depressed but the line flux is not affected, so, as a result, the equivalent width increases. B18 also finds the correlation we identify between the reflection parameter and $\Gamma$ (Figure $7(\mathrm{~g})$; $\rho=0.55 \pm 0.10 ; \log (p)=-5.31 \pm 1.78)$ but argue that it is most likely due to model-based degeneracy (see also Del Moro et al. 2017).

Figure 7(h) shows that we do not find a correlation between the intrinsic X-ray luminosity and the dominance of the AGN in the IR $(\rho=0.25 \pm 0.11 ; \log (p)=-1.41 \pm 0.92)$. This lack of a correlation suggests that our sample likely contains a range of galaxy luminosities and, by inference, black hole masses. This implies a broad range of Eddington ratios (e.g., Hopkins et al. 2009). We also do not find a correlation between the power-law 
indices of the intrinsic X-ray spectrum and its IR counterpart (Figure 7(i); $\rho=0.12 \pm 0.12 ; \log (p)=-0.47_{-0.65}^{+0.47}$ ). However, given that these two power laws trace different emission mechanisms, intrinsic coronal and reprocessed emissions, respectively, the lack of correlation is not unexpected.

The degree to which galactic-scale dust contributes to the obscuration of AGNs, and the dependence on this relative obscuration on galactic and nuclear properties, remains unclear (e.g., Rosario et al. 2012; Rovilos et al. 2012; Chen et al. 2015; Del Moro et al. 2016; Buchner \& Bauer 2017; Ricci et al. 2017b). We do not find a correlation between $N_{\mathrm{H}}$ and the SF IR luminosity from the decomposition (Figure $7(\mathrm{e}) ; \rho=0.045 \pm 0.121 ; \log (p)=-0.15_{-0.49}^{+0.15}$ ) or with the total IR luminosity $(\rho=0.070 \pm 0.122 ; \log$ $\left.(p)=-0.25_{-0.57}^{+0.25}\right)$ for our sample, indicating it is unlikely that most of the obscuration of our sources is occurring on galactic scales. Given that few of our sources (6 of 69) have $\log \left(N_{\mathrm{H}}\left[\mathrm{cm}^{-2}\right]\right) \leqslant 22$, we expect that most of our sources will require significant denser obscuration at smaller scales, consistent with the lack of correlations between IR emission associated with SF and $N_{\mathrm{H}}$. However, we cannot rule out small contributions to the obscuration from galactic scales.

\section{Appendix B \\ Investigation of Alternative Models}

To test the robustness of our conclusions regarding covering fraction distributions, we undertook the same analysis discussed in Sections 4.2.2 and 4.2.3 for five variations on our analysis. First, we used two different versions of Equation (3). We opt to retain the simple form of $\log (R) \propto f_{\text {cov }}$ but investigate the effect on our analysis if we adjust Equation (3) to have a slope of 1.4 or 2.0, with corresponding intercepts of -1.3 and -1.5 , respectively. These variations on Equation (3) are selected to still be consistent with the modeling of Baloković et al. 2018, (in preparation) but with more extreme slopes. Having determined for our original analysis that consistent results were obtained with 1000 or
10,000 bootstrap samples, we ran the 2D-KS analysis using 1000 bootstrap samples for each set of models generated with these altered empirical relations.

Figure 8 shows the equivalent to Figure 6 for variations in the empirical relation between covering fraction and reflection parameter. At flatter slopes, the models cover less of the reflection parameter range. As a result, distributions skewed to higher central values will result in better coverage of that parameter and, therefore, those models will agree better with the observations. At steeper slopes, a wider range of models, especially centered at lower covering fractions, agree with our observations. The narrowest models yielding observables similar to the data are still centered around $\sim 70 \%$. These tests demonstrate that, despite minor changes at the edges of the ranges of models that agree, the conclusion regarding the kinds of models that yield distributions of observables consistent with our data is not very sensitive to the relation between reflection parameter and covering fraction (Equation (3)).

We also examine the impact in variations in the definition of and empirical relation for IR excess. Figure 9 shows the results of these tests. First, we explored the impact of changing the dependence of the expected IR luminosity on the X-ray luminosity. Instead of using the relation from Chen et al. (2017) of $L_{\mathrm{IR}} \propto L_{\mathrm{X}}^{1 / 0.84}$, we maintained the assumption that $L_{\mathrm{IR}} \propto L_{\mathrm{bol}} \propto L_{\mathrm{UV}}$ and combined it with the relation of the UV emission to the X-ray emission of $L_{\mathrm{UV}} \propto L_{\mathrm{X}}^{1 / 0.70}$ (e.g., Steffen et al. 2006; Lusso \& Risaliti 2017). We calculated the IR excess for our observations with this change in assumption and ran the 2D-KS analysis again with this different set of measurements. The 2D-KS probabilities are uniformly lower, typically by $\sim 0.2-0.3 \mathrm{dex}$, well within the standard deviations of the probability distributions (e.g., see range of the uniform model in Figure 6). The trends regarding agreement between mock observables and the data remain constant.

Second, we relaxed the assumption of a linear scaling between IR luminosity and covering fraction. We used the IR-tobolometric luminosity dependence on covering fraction of Stalevski et al. (2016; an interpolation of the $60^{\circ}$ line of their

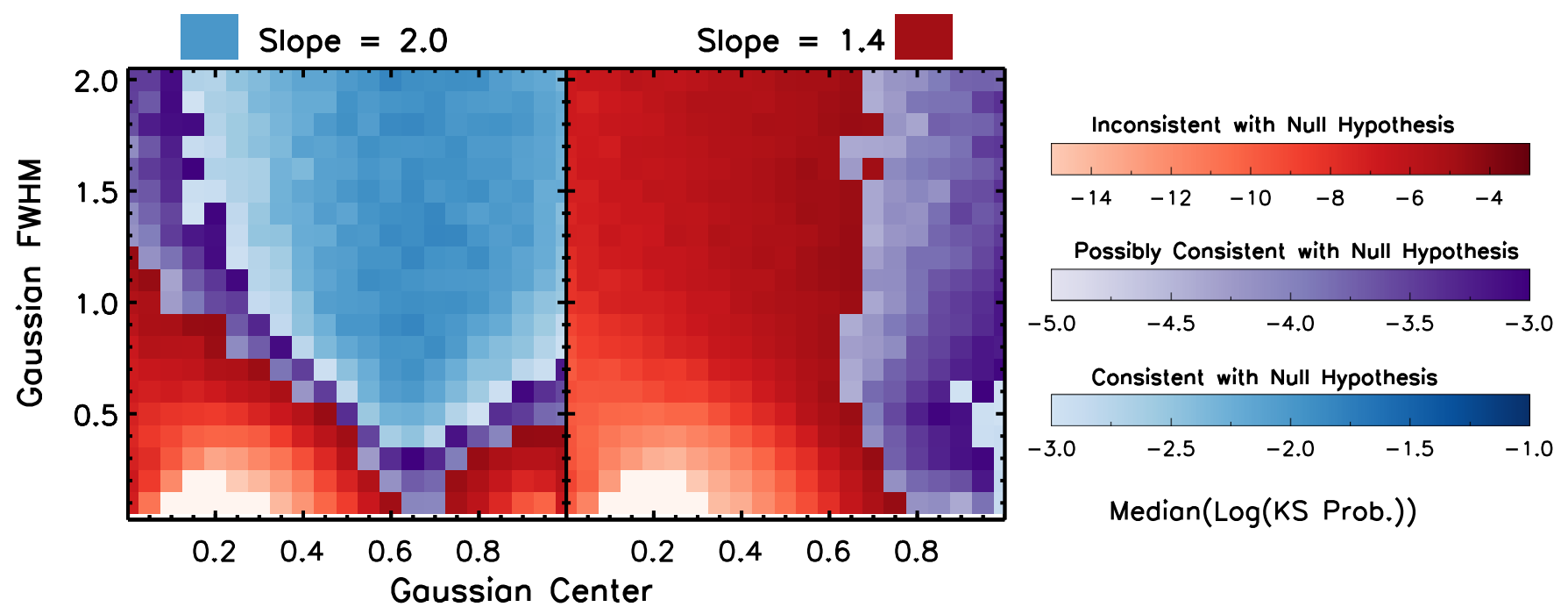

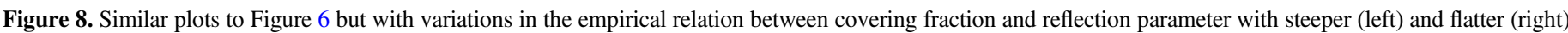

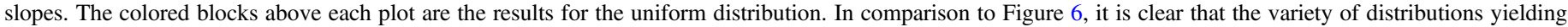

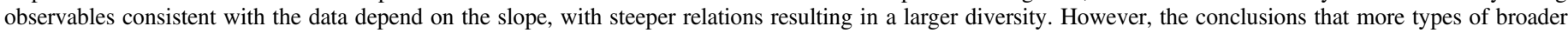

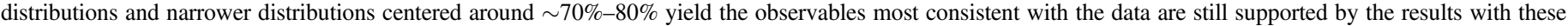
alternative relations. 


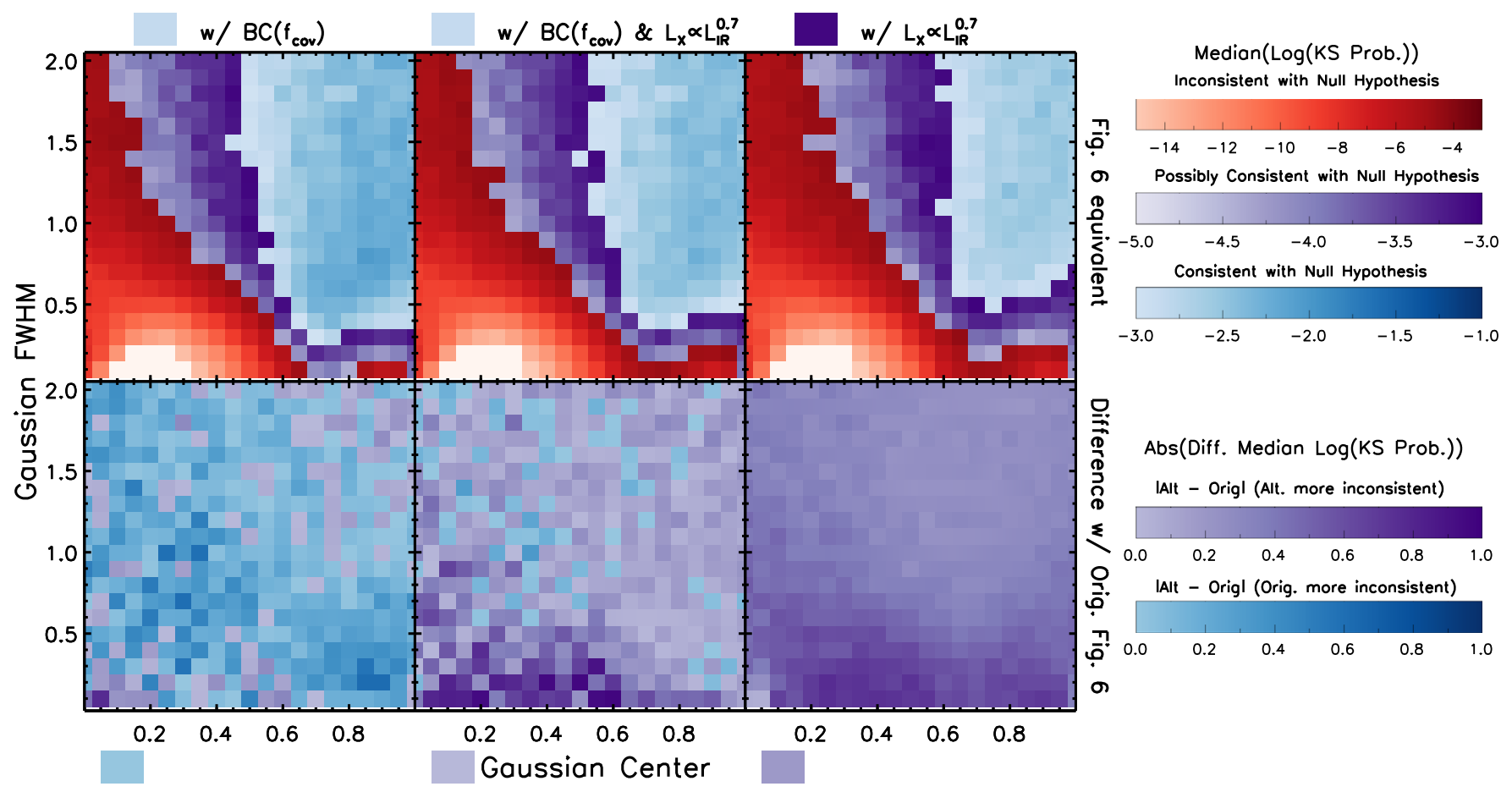

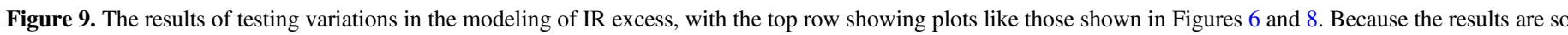

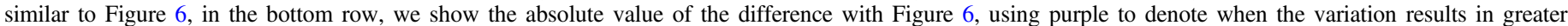

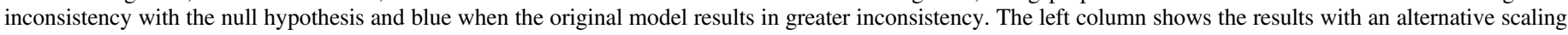

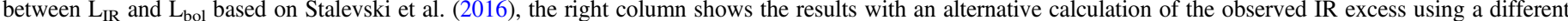

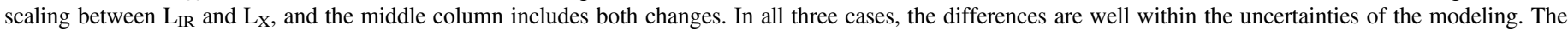
colored blocks above and below each plot show the results for the uniform distribution.

Figure 10). The relation between IR luminosity, covering fraction, and bolometric luminosity then becomes $L_{\mathrm{IR}}=\eta \times \mathrm{BC}\left(f_{\mathrm{cov}}\right) \times$ $L_{\text {bol }}$, where $\mathrm{BC}\left(f_{\text {cov }}\right)$ is the Stalevski dependence. Our Equation (4) then becomes $\log (\mathrm{IR}$ Excess $)=\log \left(\mathrm{BC}\left[\mathrm{f}_{\text {cov }}\right] /\right.$ $\left.\left\langle\mathrm{BC}\left[\mathrm{f}_{\text {cov; Type } 1}\right]\right\rangle\right)$. We ran the 2D-KS analysis using this altered empirical relation using both the original IR excess measurements and the variation described above. Adding this nonlinear dependence results in a minor improvement for many of the models at a level of $\sim 0.2-0.4$ dex, again well within the uncertainty range of the probability distribution. When both variations are put in, the changes in the probability map (i.e., Figure 6) mostly cancel out. As a result, these alterations in the definition of IR excess do not change our conclusions that broad distributions of covering fractions results in mock observables with the best agreement with our data.

\section{ORCID iDs}

Lauranne Lanz (1) https://orcid.org/0000-0002-3249-8224 Ryan C. Hickox 주 https://orcid.org/0000-0003-1468-9526 Mislav Baloković (다 https://orcid.org/0000-0003-0476-6647 Taro Shimizu (1) https://orcid.org/0000-0002-2125-4670 Claudio Ricci 이 https://orcid.org/0000-0001-5231-2645 David R. Ballantyne (1) https://orcid.org/0000-00018128-6976

Franz E. Bauer (1) https://orcid.org/0000-0002-8686-8737 Chien-Ting J. Chen (자 https://orcid.org/0000-0002-4945-5079 Duncan Farrah (1) https://orcid.org/0000-0003-1748-2010 Michael, J. Koss (1) https://orcid.org/0000-0002-7998-9581 Stephanie LaMassa (i) https://orcid.org/0000-0002-5907-3330 Alberto Masini (ib https://orcid.org/0000-0002-7100-9366 Luca Zappacosta (1) https://orcid.org/0000-0002-4205-6884

\section{References}

Alexander, D. M., \& Hickox, R. C. 2012, New Astron. Revs, 56, 93 Alonso-Herrero, A., Ramos Almeida, C., Mason, R., et al. 2011, ApJ, 736, 82 Annuar, A., Gandhi, P., Alexander, D. M., et al. 2015, ApJ, 815, 36 Antonucci, R. 1993, ARA\&A, 31, 473

Arnaud, K. A. 1996, in ASP Conf. Ser.101, Astronomical Data Analysis Software and Systems V, ed. G. H. Jacoby \& J. Barnes (San Francisco, CA: ASP), 17

Asmus, D., Gandhi, P., Hönig, S. F., Smette, A., \& Duschl, W. J. 2015, MNRAS, 454, 766

Asmus, D., Gandhi, P., Smette, A., Hönig, S. F., \& Duschl, W. J. 2011, A\&A, 536, A36

Asmus, D., Hönig, S. F., Gandhi, P., Smette, A., \& Duschl, W. J. 2014, MNRAS, 439, 1648

Baloković, M. 2017, PhD Thesis, California Institute of Technology

Baloković, M., Brightman, M., Harrison, F. A., et al. 2018, ApJ, 854, 42

Baloković, M., Comastri, A., Harrison, F. A., et al. 2014, ApJ, 794, 111

Barthelmy, S. D., Barbier, L. M., Cummings, J. R., et al. 2005, SSRv, 120, 143

Baumgartner, W. H., Tueller, J., Markwardt, C. B., et al. 2013, ApJS, 207, 19 Bell, E. F., Wolf, C., Meisenheimer, K., et al. 2004, ApJ, 608, 752

Boselli, A., Eales, S., Cortese, L., et al. 2010, PASP, 122, 261

Buchner, J., \& Bauer, F. E. 2017, MNRAS, 465, 4348

Burrows, D. N., Hill, J. E., Nousek, J. A., et al. 2005, SSRv, 120, 165

Chen, C.-T. J., Hickox, R. C., Alberts, S., et al. 2015, ApJ, 802, 50

Chen, C.-T. J., Hickox, R. C., Goulding, A. D., et al. 2017, ApJ, 837, 145

Dadina, M. 2008, A\&A, 485, 417

Dauser, T., García, J., Walton, D. J., et al. 2016, A\&A, 590, A76

Del Moro, A., Alexander, D. M., Aird, J. A., et al. 2017, ApJ, 849, 57

Del Moro, A., Alexander, D. M., Bauer, F. E., et al. 2016, MNRAS, 456, 2105

Diamond-Stanic, A. M., Rieke, G. H., \& Rigby, J. R. 2009, ApJ, 698, 623

Efstathiou, A., \& Rowan-Robinson, M. 1995, MNRAS, 273, 649

Elitzur, M. 2008, NewAR, 52, 274

Elitzur, M. 2012, ApJL, 747, L33

Esposito, V., \& Walter, R. 2016, A\&A, 590, A49

Fabian, A. C., Vasudevan, R. V., \& Gandhi, P. 2008, MNRAS, 385, L43

Farrah, D., Baloković, M., Stern, D., et al. 2016, ApJ, 831, 76

Feigelson, E. D., \& Berg, C. J. 1983, ApJ, 269, 400 
Feltre, A., Hatziminaoglou, E., Fritz, J., \& Franceschini, A. 2012, MNRAS, 426, 120

Fiore, F., Puccetti, S., Brusa, M., et al. 2009, ApJ, 693, 447

Fritz, J., Franceschini, A., \& Hatziminaoglou, E. 2006, MNRAS, 366, 767

Gandhi, P., Horst, H., Smette, A., et al. 2009, A\&A, 502, 457

Gandhi, P., Lansbury, G. B., Alexander, D. M., et al. 2014, ApJ, 792, 117

Gehrels, N., Chincarini, G., Giommi, P., et al. 2004, ApJ, 611, 1005

George, I. M., \& Fabian, A. C. 1991, MNRAS, 249, 352

Goulding, A. D., Forman, W. R., Hickox, R. C., et al. 2014, ApJ, 783, 40

Griffin, M. J., Abergel, A., Abreu, A., et al. 2010, A\&A, 518, L3

Guilbert, P. W., \& Rees, M. J. 1988, MNRAS, 233, 475

Gültekin, K., Richstone, D. O., Gebhardt, K., et al. 2009, ApJ, 698, 198

Harrison, F. A., Craig, W. W., Christensen, F. E., et al. 2013, ApJ, 770, 103

Hernández-García, L., Masegosa, J., González-Martín, O., et al. 2017, A\&A, 602, A65

Hickox, R. C., \& Alexander, D. M. 2018, ARA\&A, 56, 625

Hönig, S. F., \& Kishimoto, M. 2010, A\&A, 523, A27

Hönig, S. F., Kishimoto, M., Antonucci, R., et al. 2012, ApJ, 755, 149

Hopkins, P. F., Hickox, R., Quataert, E., \& Hernquist, L. 2009, MNRAS, 398,333

Ichikawa, K., Packham, C., Ramos Almeida, C., et al. 2015, ApJ, 803, 57

Ichikawa, K., Ricci, C., Ueda, Y., et al. 2017, ApJ, 835, 74

Ichikawa, K., Ricci, C., Ueda, Y., et al. 2018, arXiv:1811.02568

Ichikawa, K., Ueda, Y., Terashima, Y., et al. 2012, ApJ, 754, 45

Isobe, T., Feigelson, E. D., \& Nelson, P. I. 1986, ApJ, 306, 490

Kormendy, J., \& Ho, L. C. 2013, ARA\&A, 51, 511

Koss, M., Trakhtenbrot, B., Ricci, C., et al. 2017, ApJ, 850, 74

Koss, M. J., Assef, R., Baloković, M., et al. 2016, ApJ, 825, 85

Krolik, J. H., \& Begelman, M. C. 1986, ApJL, 308, L55

Lamperti, I., Koss, M., Trakhtenbrot, B., et al. 2017, MNRAS, 467, 540

Lavalley, M., Isobe, T., \& Feigelson, E. 1992, in ASP Conf. Ser.25, Astronomical Data Analysis Software and Systems I, ed. D. M. Worrall, C. Biemesderfer, \& J. Barnes (San Francisco, CA: ASP), 245

Lawrence, A. 1991, MNRAS, 252, 586

Levenson, N. A., Heckman, T. M., Krolik, J. H., Weaver, K. A., \& Życki, P. T. 2006, ApJ, 648, 111

López-Gonzaga, N., Burtscher, L., Tristram, K. R. W., Meisenheimer, K., \& Schartmann, M. 2016, A\&A, 591, A47

Lusso, E., \& Risaliti, G. 2017, A\&A, 602, A79

Lutz, D., Maiolino, R., Spoon, H. W. W., \& Moorwood, A. F. M. 2004, A\&A, 418, 465

Madau, P., Ghisellini, G., \& Fabian, A. C. 1993, ApJL, 410, L7

Magdziarz, P., \& Zdziarski, A. A. 1995, MNRAS, 273, 837

Maiolino, R., Shemmer, O., Imanishi, M., et al. 2007, A\&A, 468, 979

Marconi, A., Risaliti, G., Gilli, R., et al. 2004, MNRAS, 351, 169

Markwardt, C. B. 2009, in ASP Conf. Ser.411, Astronomical Data Analysis Software and Systems XVIII, ed. D. A. Bohlender, D. Durand, \& P. Dowler (San Francisco, CA: ASP), 251

Mateos, S., Carrera, F. J., Alonso-Herrero, A., et al. 2016, ApJ, 819, 166

Mateos, S., Carrera, F. J., Barcons, X., et al. 2017, ApJL, 841, L18

Matsuta, K., Gandhi, P., Dotani, T., et al. 2012, ApJ, 753, 104

Matt, G., \& Fabian, A. C. 1994, MNRAS, 267, 187

McConnell, N. J., \& Ma, C.-P. 2013, ApJ, 764, 184

Meléndez, M., Mushotzky, R. F., Shimizu, T. T., Barger, A. J., \& Cowie, L. L. 2014, ApJ, 794, 152

Merloni, A., \& Heinz, S. 2008, MNRAS, 388, 1011
Mor, R., Netzer, H., \& Elitzur, M. 2009, ApJ, 705, 298

More, J. J. 1978, in Lecture Notes in Mathematics 630: Numerical Analysis, ed. G. A. Watson (Berlin: Springer-Verlag), 105

Mullaney, J. R., Alexander, D. M., Goulding, A. D., \& Hickox, R. C. 2011, MNRAS, 414, 1082

Murphy, K. D., \& Yaqoob, T. 2009, MNRAS, 397, 1549

Mushotzky, R. F., Winter, L. M., McIntosh, D. H., \& Tueller, J. 2008, ApJL, 684, L65

Nandra, K., George, I. M., Mushotzky, R. F., Turner, T. J., \& Yaqoob, T. 1997, ApJL, 488, L91

Nenkova, M., Sirocky, M. M., Nikutta, R., Ivezić, Ž., \& Elitzur, M. 2008, ApJ, 685,160

Netzer, H. 2015, ARA\&A, 53, 365

Nikutta, R., Elitzur, M., \& Lacy, M. 2009, ApJ, 707, 1550

Oppenheimer, B. D., Davé, R., Kereš, D., et al. 2010, MNRAS, 406, 2325

Padovani, P., Alexander, D. M., Assef, R. J., et al. 2017, A\&ARv, 25, 2

Peacock, J. A. 1983, MNRAS, 202, 615

Piconcelli, E., Jimenez-Bailón, E., Guainazzi, M., et al. 2005, A\&A, 432, 15

Pier, E. A., \& Krolik, J. H. 1993, ApJ, 418, 673

Pihajoki, P. 2017, MNRAS, 472, 3407

Poglitsch, A., Waelkens, C., Geis, N., et al. 2010, A\&A, 518, L2

Ramos Almeida, C., Levenson, N. A., Alonso-Herrero, A., et al. 2011, ApJ, 731,92

Reeves, J. 2003, in ASP Conf. Ser.290, Active Galactic Nuclei: From Central Engine to Host Galaxy, ed. S. Collin, F. Combes, \& I. Shlosman (San Francisco, CA: ASP), 35

Ricci, C., Trakhtenbrot, B., Koss, M. J., et al. 2017a, ApJS, 233, 17

Ricci, C., Trakhtenbrot, B., Koss, M. J., et al. 2017b, Natur, 549, 488

Ricci, C., Ueda, Y., Koss, M. J., et al. 2015, ApJL, 815, L13

Ricci, C., Walter, R., Courvoisier, T. J.-L., \& Paltani, S. 2011, A\&A, 532, A102

Rigby, J. R., Diamond-Stanic, A. M., \& Aniano, G. 2009, ApJ, 700, 1878

Rosario, D. J., Santini, P., Lutz, D., et al. 2012, A\&A, 545, A45

Rovilos, E., Comastri, A., Gilli, R., et al. 2012, A\&A, 546, A58

Shimizu, T. T., Meléndez, M., Mushotzky, R. F., et al. 2016, MNRAS, 456, 3335

Shimizu, T. T., Mushotzky, R. F., Meléndez, M., et al. 2017, MNRAS, 466, 3161

Siebenmorgen, R., Heymann, F., \& Efstathiou, A. 2015, A\&A, 583, A120

Simpson, C. 2005, MNRAS, 360, 565

Spergel, D. N., Bean, R., Doré, O., et al. 2007, ApJS, 170, 377

Stalevski, M., Fritz, J., Baes, M., Nakos, T., \& Popović, L. Č. 2012, MNRAS, 420, 2756

Stalevski, M., Ricci, C., Ueda, Y., et al. 2016, MNRAS, 458, 2288

Steffen, A. T., Strateva, I., Brandt, W. N., et al. 2006, AJ, 131, 2826

Stern, J., \& Laor, A. 2012, MNRAS, 426, 2703

Treister, E., Krolik, J. H., \& Dullemond, C. 2008, ApJ, 679, 140

Vasudevan, R. V., Fabian, A. C., Gandhi, P., Winter, L. M., \& Mushotzky, R. F. 2010, MNRAS, 402, 1081

Vasudevan, R. V., Mushotzky, R. F., \& Gandhi, P. 2013, ApJL, 770, L37

Winter, L. M., Mushotzky, R. F., Reynolds, C. S., \& Tueller, J. 2009, ApJ, 690, 1322

Wright, E. L., Eisenhardt, P. R. M., Mainzer, A. K., et al. 2010, AJ, 140, 1868

Xie, F.-G., \& Yuan, F. 2017, ApJ, 836, 104

Yaqoob, T., \& Murphy, K. D. 2011, MNRAS, 412, 835 